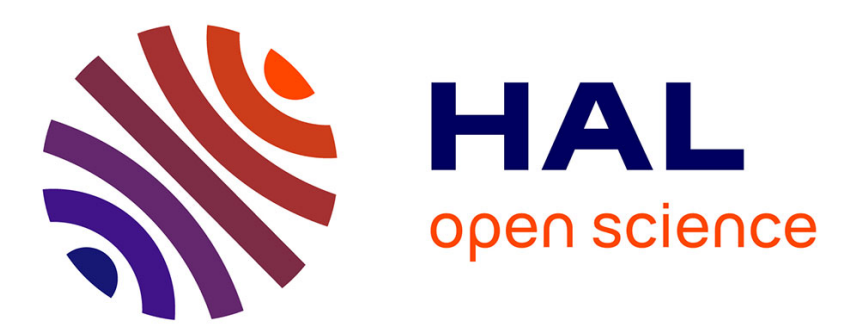

\title{
Time-optimal control of a particle in a dielectrophoretic system
}

Dong Eui Chang, Nicolas Petit, Pierre Rouchon

\section{To cite this version:}

Dong Eui Chang, Nicolas Petit, Pierre Rouchon. Time-optimal control of a particle in a dielectrophoretic system. IEEE Transactions on Automatic Control, 2006, 51 (7), pp.100-1114. 10.1109/TAC.2006.878748 . hal-00531113

HAL Id: hal-00531113

https://hal-mines-paristech.archives-ouvertes.fr/hal-00531113

Submitted on 1 Nov 2010

HAL is a multi-disciplinary open access archive for the deposit and dissemination of scientific research documents, whether they are published or not. The documents may come from teaching and research institutions in France or abroad, or from public or private research centers.
L'archive ouverte pluridisciplinaire HAL, est destinée au dépôt et à la diffusion de documents scientifiques de niveau recherche, publiés ou non, émanant des établissements d'enseignement et de recherche français ou étrangers, des laboratoires publics ou privés. 


\title{
Time-Optimal Control of a Particle in a Dielectrophoretic System
}

\author{
Dong Eui Chang, Nicolas Petit, and Pierre Rouchon
}

\begin{abstract}
We study the time-optimal control of a particle in a dielectrophoretic system. This system consists of a time-varying nonuniform electric field which acts upon the particle by creating a dipole within it. The interaction between the induced dipole and the electric field generates the motion of the particle. The control is the voltage on the electrodes which induces the electric field. Since we are considering the motion of a particle on an invariant line in a chamber filled with fluid flowing at low Reynolds number, the dynamics have a two dimensional state; one for the particle position and the other for the induced dipole moment. In regard to time-optimal control, we address the issue of existence and uniqueness of optimal trajectories, and explicitly compute the optimal control and the corresponding minimum time. Finally, we cast our analysis in the framework of symplectic reduction theory in order to provide geometric insight into the problem.
\end{abstract}

Index Terms-Biotechnology, dielectrophoresis, nanotechnology, time-optimal control.

\section{INTRODUCTION}

$\mathbf{W}$ E STUDY the time-optimal control of the following system:

$$
\begin{aligned}
& \dot{x}=y u+\alpha u^{2} \\
& \dot{y}=-c y+u
\end{aligned}
$$

with the state $(x, y) \in \mathbb{R}^{2}$ and the single control $u$ satisfying

$$
\begin{aligned}
x(0) & =x_{0}=\text { given } & & y(0)=0 \\
x\left(t_{f}\right) & =x_{f}=\text { given } & & y\left(t_{f}\right)=\text { free } \\
|u| & \leq 1 & &
\end{aligned}
$$

where the parameters $\alpha$ and $c$ satisfy

$$
\alpha<0 \quad c>0
$$

These dynamics describe, after a nonlinear change of coordinates, the motion of a neutrally buoyant and neutrally charged particle on an invariant line in a chamber filled with fluid flowing at low Reynolds number and with a parallel electrode array at the bottom of the chamber. The existence of the invariant line is due to symmetry in the arrangement of electrodes and the boundary potential on electrodes. The motion is created by the interaction between a nonuniform electric field and the dipole moment

Manuscript received January 31, 2005; revised November 21, 2005. Recommended by Associate Editor U. Jonsson.

D. E. Chang is with the Department of Applied Mathematics, Faculty of Mathematics, the University of Waterloo, Waterloo, ON N2L 3G1, Canada.

N. Petit and P. Rouchon are with the Centre Automatique et Systèmes, the Ecole Nationale Supérieure des Mines de Paris, Paris 75272, France.

Digital Object Identifier 10.1109/TAC.2006.878748 induced in the particle. This motion is called dielectrophoresis (DEP) [11]. Dielectrophoresis has wide applications in nano/ bio-technology, in particular, in manipulating, separating and identifying nano/bio-particles [7], [8].

A brief explanation of the dynamics is in order. The variable $x$ describes the displacement of the particle. The variable $y$ describes the exponentially decaying part of the induced dipole moment. Voltage $u$ is given on every other electrode and $(-u)$ on the others. Parameters $\alpha$ and $c$ depend on the permittivities and conductivities of the particle and the fluid medium. The positivity of $c$ is imposed by physics, but the negativity of $\alpha$ is arbitrary. As one is not interested in the final value of the induced dipole moment, the final value of $y$ is free in (4).

Our goal in this paper is to study the minimum time trajectories of this system. A complete solution of this problem in a general setup-for a set of particles in a three-dimensional space, for instance-would allow significant improvement in DEP-based devices for particle analysis such as detecting cancers cells and separating different cells. However, here we address a simple case, which still includes the key feature of dielectrophoresis. Various control problems on dielectrophoresis in nano/biotechnology are suggested in [5].

This paper is organized as follows. We first overview the main results of the paper. Second, we derive the dynamics from physics. Third, we study the nonexistence of Lebesgue measurable time-optimal control for $x_{f}<x_{0}$ even though the target point is reachable. Fourth, we show that $(1+\alpha c)>0$ is the necessary and sufficient condition, under assumption (6), for the existence of time-optimal controls when $x_{f}>x_{0}$. Fifth, we address the issue of uniqueness of time-optimal control when $x_{f}>x_{0}$. We find a condition on $\alpha$ and $c$ which guarantees the uniqueness of optimal trajectories, and compute the minimum time and the optimal control for a given target point $x_{f}$. For the case that the uniqueness condition is not satisfied, we give a constructive algorithm with which we can easily find a time-optimal control. Sixth, we make a discussion on the case where the optimal trajectories derived above are still valid in the presence of a state constraint on $x$ such as $x \geq 0$ or $x \leq 0$. Seventh, we give geometric insight into the problem by putting the previous analysis in the picture of symplectic reduction theory. Finally, we perform some simulations to demonstrate the result.

\section{OVERVIEW OF MAIN RESUlts}

Time optimal trajectories satisfy the following dynamics:

$$
\begin{aligned}
& \dot{x}=y u+\alpha u^{2} \\
& \dot{y}=-c y+u \\
& \dot{\lambda}=c \lambda-u
\end{aligned}
$$


with conditions (3)-(6) and

$$
\lambda(0)=\lambda_{0}=\text { to be found } \quad \lambda\left(t_{f}\right)=0
$$

where

$$
u(t)=\arg \max _{|v| \leq 1} \tilde{H}(y(t), \lambda(t), v)
$$

with

$$
\tilde{H}(y, \lambda, v)=\alpha u^{2}+(y+\lambda) u-c y \lambda .
$$

The control $u$ satisfying (11) is given by

$$
u= \begin{cases}+1, & \text { if } y+\lambda>-2 \alpha \\ \frac{y+\lambda}{-2 \alpha}, & \text { if }|y+\lambda| \leq-2 \alpha \\ -1, & \text { if } y+\lambda<2 \alpha\end{cases}
$$

because $\alpha<0$. A symplectic reduction picture is hidden in (7)-(12).

When $x_{f}<x_{0}$, there are no Lebesgue measurable time-optimal control functions resulting in $x\left(t_{f}\right)=x_{f}$ even though $x_{f}$ is reachable.

We now consider the case of $x_{f}>x_{0}$. Time-optimal control law exists if and only if the parameters, $\alpha$ and $c$ satisfy $(1+$ $\alpha c)>0$, which is assumed in the following of this section. Define the open interval $\Lambda$ by

$$
\Lambda=(-2 \alpha \sqrt{1+\alpha c},-2 \alpha \sqrt{(1+\alpha c) /(-\alpha c)})
$$

if $(1+2 \alpha c) \leq 0$, and

$$
\begin{aligned}
\Lambda & =\Lambda_{1} \cup \Lambda_{2} \\
& =(-2 \alpha \sqrt{1+\alpha c},-2 \alpha) \cup[-2 \alpha, 1 / c)
\end{aligned}
$$

if $(1+2 \alpha c)>0$. For the sake of convenience, let us define four sentences as follows:

- $\mathrm{P} 1:=[(1+2 \alpha c)>0] \wedge\left[\lambda_{0} \in \Lambda_{1}\right]$

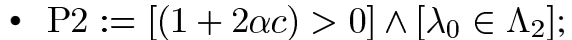

- $\mathrm{Q}:=[(1+2 \alpha c) \leq 0] \wedge\left[\lambda_{0} \in \Lambda\right]$

- $\mathrm{R}:=[(3+4 \alpha c) \geq 0] \wedge\left[\lambda_{0} \in \Lambda\right]$

where $\wedge$ is the logical connective AND. Let us define a strictly increasing onto function $X_{1}: \Lambda \rightarrow(0, \infty)$ by

$$
X_{1}\left(\lambda_{0}\right)= \begin{cases}\text { equation }(63), & \text { if } \mathrm{P} 1 \vee \mathrm{Q} \\ \text { equation }(67), & \text { if } \mathrm{P} 2\end{cases}
$$

where $\vee$ is the logical connective OR. Let us define another function $T_{1}$ on $\Lambda$ by

$$
T_{1}\left(\lambda_{0}\right)= \begin{cases}\text { equation (73), } & \text { if } \mathrm{P} 1 \vee \mathrm{Q} \\ \text { equation (74), } & \text { if } \mathrm{P} 2 .\end{cases}
$$

We call a trajectory of (7)-(9) satisfying (3)-(5) and (10)-(11), an extremal. Let us call an arc of an extremal a basic arc if the projection of the arc onto the $y-\lambda$ plane starts from
$0 \times \Lambda$ (respectively, $0 \times(-\Lambda)$ ) and ends on $\Lambda \times 0$ (respectively, $(-\Lambda) \times 0$ ), going through the first (respectively, third) quadrant of the $y-\lambda$ plane. We call an extremal an $n$-shot extremal with $n \in \mathbb{N}$ if the maximum number of basic arcs in the extremal is $n$.

To decompose extremals into finite $\operatorname{arcs}$ when $[\mathrm{P} 1 \vee \mathrm{Q}]$, let us introduce some notation. An arc associated with the linear control $u=(y+\lambda) /(-2 \alpha)$ on a time interval of length $\Delta t_{A B}\left(\lambda_{0}\right)$ in (71), is denoted by $\gamma_{\mathrm{L}}^{\lambda_{0}}$. Let $\gamma_{+}^{\lambda_{0}}$ (respectively, $\gamma_{-}^{\lambda_{0}}$ ) denote an arc with $u=1$ (respectively, $u=-1$ ) on a time interval of length $\Delta t_{B C}\left(\lambda_{0}\right)$ in (72). Define two arcs $\Gamma_{ \pm}^{\lambda_{0}}$ by the concatenation

$$
\Gamma_{ \pm}^{\lambda_{0}}=\gamma_{L}^{\lambda_{0}} \star \gamma_{ \pm}^{\lambda_{0}} \star \gamma_{L}^{\lambda_{0}}
$$

where the concatenation $\star$ is defined such that the leftmost one comes first and the rightmost one comes last. An arc with the linear control $u=(y+\lambda) /(-2 \alpha)$ is called an idling arc if its projection $(y, \lambda)$ starts from the positive (respectively, negative) $y$-axis, goes through the fourth (respectively, second) quadrant in the $y-\lambda$ plane, and finally ends at the negative (respectively, positive) $\lambda$-axis. An idling arc is denoted by $\gamma_{\text {idling }}$; see Fig. 8 . Its duration $T_{\text {idling }}$ is given in (76). Hence, when $[\mathrm{P} 1 \vee \mathrm{Q}]$, we can express $n$-shot extremals as $\Gamma_{ \pm}^{\lambda_{0}, n}$ for $n \in \mathbb{N}$, which are defined as follows:

$$
\begin{aligned}
& \Gamma_{ \pm}^{\lambda_{0}, 1}=\Gamma_{ \pm}^{\lambda_{0}} \\
& \Gamma_{ \pm}^{\lambda_{0}, k}= \begin{cases}\Gamma_{ \pm}^{\lambda_{0}, k-1} \star \gamma_{\text {idling }} \star \Gamma_{\mp}^{\lambda_{0}}, & \text { if } k \text { is even } \\
\Gamma_{ \pm}^{\lambda_{0}, k-1} \star \gamma_{\text {idling }} \star \Gamma_{ \pm}^{\lambda_{0}}, & \text { if } k \text { is odd }\end{cases}
\end{aligned}
$$

for $k \geq 2$ with

$$
\lambda(0)= \begin{cases}+\lambda_{0}, & \text { for } \Gamma_{+}^{\lambda_{0}, m} \\ -\lambda_{0}, & \text { for } \Gamma_{-}^{\lambda_{0}, m}\end{cases}
$$

for $m \geq 1$.

We now discuss the existence and uniqueness of optimal trajectories. If $(3+4 \alpha c) \geq 0$, there exist exactly two time-optimal trajectories for $x_{f}>x_{0}$, and they are basic arcs. Here is the procedure of constructing them.

[A.1.] Find $\lambda_{0}=X_{1}^{-1}\left(x_{f}-x_{0}\right) \in \Lambda$.

[A.2.] Set $\lambda(0)= \pm \lambda_{0}$.

[A.3.] The minimum time cost is $T_{1}\left(\lambda_{0}\right)$ and the optimal trajectories $\gamma_{\mathrm{opt}}$ are

$$
\gamma_{\text {opt }}= \begin{cases}\Gamma_{ \pm}^{\lambda_{0}}, & \text { if } \mathrm{R} \wedge \neg \mathrm{P} 2 \\ \text { basic arc with } u= \pm 1, & \text { if } \mathrm{P} 2\end{cases}
$$

where $\neg$ is the logical connective NOT.

If $(3+4 \alpha c)<0$, we do not have any general proof of the uniqueness of optimal control. However, we have a finite procedure of finding all optimal control laws for $x_{f}>x_{0}$ as follows.

[B.1.] Define two sequences, for $k \in \mathbb{N}$

$$
\begin{aligned}
\lambda_{0, k} & =X_{1}^{-1}\left(\frac{x_{f}-x_{0}}{k}\right) \\
T_{k} & =k T_{1}\left(\lambda_{0, k}\right)+(k-1) T_{\text {idling. }} .
\end{aligned}
$$




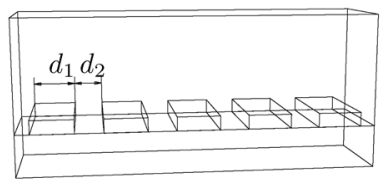

(a)

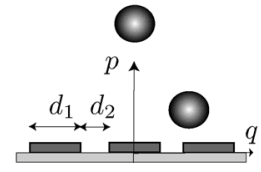

(b)
Fig. 1. Dielectrophoretic system is a chamber filled with a fluid medium where there is a parallel array of electrodes at the bottom.

[B.2.] Find $n=\arg \min _{k}\left\{T_{k}: k \in \mathbb{N}\right\}$ (such an $n$ always exists and is less than $1+\left(T_{1} / T_{\text {idling }}\right)$ ).

[B.3.] Set $\lambda(0)= \pm \lambda_{0, n}$.

[B.4.] The minimum time cost is $T_{n}$ and the corresponding optimal trajectories are $\Gamma_{ \pm}^{\lambda_{0}, n}$.

If there are $j$ integers in step [B.2.] to give the minimum time, then there are exactly $2 j$ time-optimal trajectories. We remark that each basic arc in the $k$-shot extremal $\Gamma_{ \pm}^{\lambda_{0, k}, k}$ equally contributes $\left(x_{f}-x_{0}\right) / k$ to the increment of $x$ and the idling arcs in between make no contribution.

In Section VI-H, we discuss the possibility that some optimal trajectories derived previously become optimal even with state constraints $x \geq 0$ or $x \leq 0$. In Section VII, we draw a symplectic reduction picture hidden in the problem and our solution.

\section{DERIVATION OF DYNAMICS}

We briefly derive the dynamics in (1) and (2), and explain the conditions in (3)-(6); see [4] for more details. Consider a neutrally charged particle in a chamber with a fluid medium and a parallel electrode array at the bottom as in Fig. 1(a) where $d_{1}$ is the width of each electrode, and $d_{2}$ is the width of the gap between two electrodes. As the electrodes are very long compared with the size of particles, we may assume that there are infinite number of infinitely long electrodes. Due to this symmetry, we can consider the motion of the particle in the vertical plane as in Fig. 1(b).

Let $(q, p) \in \mathbb{R}^{2}$ be the coordinates in Fig. 1(b). We give the boundary voltage

$$
V_{\mathrm{bd}}(t)=V_{0} \cdot u(t), \quad|u(t)| \leq 1
$$

on every other electrode and $\left(-V_{\mathrm{bd}}(t)\right)$ on the others. This creates potential $V(q, p, t)$ in $\{p \geq 0\}$. The electric field vector $\mathbf{E}(q, p, t) \in \mathbb{R}^{2}$ in $\{p \geq 0\}$ is given by

$$
\mathbf{E}(q, p, t)=-\nabla V(q, p, t) .
$$

This electric field induces a dipole moment $\mathbf{m}$ in a single-layered spherical particle as follows:

$$
\mathbf{m}(q, p, t)=g(t) * \mathbf{E}(q, p, t)
$$

where $*$ denotes the usual convolution operator with respect to time $t$ and the Laplace transform $G(s)$ of the (transfer) function $g(t)$ is given by

$$
G(s)=a+\frac{b}{s+c}
$$

where

$$
\begin{aligned}
a & =4 \pi r^{3} \epsilon_{m}\left(\epsilon_{p}-\epsilon_{m}\right) /\left(\epsilon_{p}+2 \epsilon_{m}\right) \\
b & =a\left(\frac{\sigma_{p}-\sigma_{m}}{\epsilon_{p}-\epsilon_{m}}-\frac{\sigma_{p}+2 \sigma_{m}}{\epsilon_{p}+2 \epsilon_{m}}\right) \\
c & =\left(\sigma_{p}+2 \sigma_{m}\right) /\left(\epsilon_{p}+2 \epsilon_{m}\right)
\end{aligned}
$$

where $r$ is the radius of the particle, $\epsilon_{p}$ (respectively, $\epsilon_{m}$ ) is the permittivity of the particle (resp., medium) and $\sigma_{p}$ (respectively, $\sigma_{m}$ ) is the conductivity of the particle (respectively, medium). The interaction between the electric field and the induced dipole moment creates a force $\mathbf{F}_{\mathrm{dep}}$. It is called dielectrophoretic force and is given by

$$
\mathbf{F}_{\mathrm{dep}}(q, p, t)=(\mathbf{m}(q, p, t) \cdot \nabla) \mathbf{E}(q, p, t) .
$$

We restrict our interest to the motion of a particle on the $p$-axis because it can practically represent the vertical motion of all particles in the whole chamber. One can check that the dielectrophoretic force on the $p$-axis is parallel to this axis due to the symmetry in the boundary voltage. This vertical dielectrophoretic force on the $p$-axis is denoted by $F_{\text {dep }}(p, t)$. It is of the form

$$
F_{\text {dep }}(p, t)=F(p) u(t)(g * u)(t)
$$

where

$$
F(p)=\frac{-\pi^{3} V_{0}^{2} e^{-\frac{\pi p}{2 d}}\left(1-e^{-\frac{\pi p}{d}}\right)}{2 d^{3} K^{2}\left(\cos \left(\frac{d_{2} \pi}{4 d}\right)\right)\left[1-2 e^{-\frac{\pi p}{2 d}} \cos \left(\frac{d_{1} \pi}{2 d}\right)+e^{-\frac{\pi p}{d}}\right]^{2}}
$$

where $K$ is the complete elliptic function of the first kind and $d=\left(d_{1}+d_{2}\right) / 2$; see [4] for the derivation of $F$. Notice that $F(p) \leq 0$ on $p \geq 0, \lim _{p \rightarrow \infty} F(p)=0$, and $F(p)=0$ only at $p=0$.

Let us assume that the particle is neutrally buoyant and the medium fluid flows at low Reynolds number. Thus, the gravitational force and the buoyant force cancel and the inertial term $m \ddot{p}$ is trivial. The only forces on the particle are the drag and the DEP force. Hence, the motion of the particle on the $p$-axis can be described by

$$
f \dot{p}+F(p) u(t)(g * u)(t)=0 .
$$

where $f>0$ is the drag constant.

We assume that $b$ in (20) is nonzero, which generically holds. Then, (1) and (2) come from (22) where $x$ and $y$ are defined by

$$
\begin{aligned}
x & =\int_{\epsilon}^{p} \frac{-f}{b F(z)} \mathrm{d} z \\
Y(s) & =\frac{1}{s+c} U(s)
\end{aligned}
$$


for $p \geq \epsilon$ where $\epsilon$ is a positive number and $Y(s)$ and $U(s)$ are the Laplace transforms of $y(t)$ and $u(t)$, and $\alpha$ is defined by

$$
\alpha=a / b \text {. }
$$

If a particle is close to the electrode, then additional physical/ chemical forces other than the DEP force start to appear in the dynamics [7], [8], [11], so the parameter $\epsilon$ in (23) defines the region where the dynamics (22) is valid. Physically, $y$ is the exponentially induced part of the dipole moment, so we have the initial condition $y(0)=0$. As we are not interested in the final state of the induced dipole moment, we have $y\left(t_{f}\right)=$ free.

Depending on the sign of $b$, the original region $\{p \geq \epsilon\}$ is mapped to $\{x \geq 0\}$ or $\{x \leq 0\}$. In this paper, we ignore this state constraint on $x$, allowing for $x$ to be on the whole real line. In Section VI-H, we discuss the possibility that the time optimal trajectories without the state constraints remain optimal with the state constraints. In a future publication, we will address the optimization problem with the state constraint on $x$.

We also make the following assumption on the signs of parameters $\alpha$ and $c$

$$
\alpha<0 \quad c>0 .
$$

The assumption $c>0$ is imposed by physics; see (21). However, the condition $\alpha<0$ is chosen for convenience. The case where $\alpha \geq 0$ is left for future work.

\section{NONEXISTENCE OF OPTIMAL CONTROL FOR $x_{f}<x_{0}$}

We will show that there are no time-optimal (Lebesque) measurable control functions for $x_{f}<x_{0}$ even though $x_{f}$ is reachable.

Fix a $T>0$. Let us define a sequence of functions $\left\{u_{n}^{T}\right.$ : $[0, T] \rightarrow \pm 1\}_{n \in \mathbb{N}}$ as follows:

$$
u_{n}^{T}(t)=\operatorname{sign}(\sin (2 \pi n t / T)) .
$$

It is straightforward to prove the following lemma.

Lemma 4.1: For any continuous function $f$ on $[0, T]$

$$
\lim _{n \mapsto \infty} \int_{0}^{t} f \cdot u_{n}^{T}=0
$$

uniformly in $t \in[0, T]$.

The substitution of $u$ in (2) to (1) yields

$$
\dot{x}=y \dot{y}+c y^{2}+\alpha u^{2} .
$$

It follows that

$$
\begin{aligned}
x(T) & -x(0) \\
= & \int_{0}^{T} \dot{x} d t \\
= & \frac{1}{2} y(T)^{2}+c \int_{0}^{T} y(\tau)^{2} d \tau+\alpha \int_{0}^{T} u(\tau)^{2} d \tau
\end{aligned}
$$

$$
\begin{aligned}
& \geq \alpha \int_{0}^{T} u(\tau)^{2} d \tau \\
& \geq \alpha T
\end{aligned}
$$

because $\alpha<0, x(0)=0$, and $|u| \leq 1$. This implies that $x(0)+$ $\alpha T$ is a lower bound of $x(T)$ for any admissible control $u$. Let $\left(x_{n}(t), y_{n}(t)\right), 0 \leq t \leq T$ be the solution to (1) and (2) with control $u_{n}^{T}$ in (24). In particular

$$
y_{n}(t)=e^{-c t} \int_{0}^{t} e^{c \tau} u_{n}^{T}(\tau) d \tau .
$$

Given $\epsilon>0$, by Lemma 4.1, there exists $N \in \mathbb{N}$ such that

$$
\left|y_{n}(t)\right| \leq \sqrt{\epsilon}
$$

for all $t \in[0, T]$ and all $n \geq N$. By (26), (29) and the definition of $u_{n}^{T}$, we have

$$
\left|x_{n}(T)-x(0)-\alpha T\right| \leq\left(\frac{1}{2}+c T\right) \epsilon
$$

for all $n>N$. Hence

$$
\lim _{n \rightarrow \infty} x_{n}(T)=x(0)+\alpha T .
$$

We have constructed a sequence $\left\{u_{n}^{T}\right\}$ of control laws such that the corresponding $\left\{x_{n}(T)\right\}$ converges to the lower bound $x(0)+\alpha T$ of the reachable point of $x$ in time $T$. Notice that the sequence of functions $\left\{u_{n}^{T}\right\}$ does not converge to a measurable function. The following lemma addresses this issue.

Lemma 4.4: For a given $T>0$, there exists no measurable control function $u:[0, T] \rightarrow[-1,1]$ such that $x(T)=x(0)+$ $\alpha T$.

Proof: The proof is by contradiction. Suppose that there is a measurable function $u:[0, T] \rightarrow[-1,1]$ such that $x(T)=$ $x(0)+\alpha T$. By (27), it follows that

$$
\begin{aligned}
& y(t)=0 \quad \text { a.e. on }[0, T] \\
& u(t)= \pm 1 \text { a.e. on }[0, T] .
\end{aligned}
$$

Hence, for almost all $t \in[0, T]$

$$
\begin{aligned}
0 & =y(t)=\int_{0}^{t} \dot{y}(s) d s \\
& =\int_{0}^{t}(-c y(s)+u(s)) d s=\int_{0}^{t} u(s) d s .
\end{aligned}
$$

By [6, Th. 4.9], the function $t \mapsto \int_{0}^{t} u(s) d s$ is continuous. It follows that

$$
\int_{0}^{t} u(s) d s=0
$$

for all $t \in[0, T]$. By $[6,4.11], u(t)=0$ for almost all $t \in[0, T]$ which contradicts (31). Thus, there exists no such measurable function $u$ that produces $x(T)=x(0)+\alpha T$. 
We have shown the following.

Claim 4.3: For $x_{f}<x_{0}$, the infimum of the time cost is $T=\left(x_{0}-x_{f}\right) /(-\alpha)$, but there are no time-optimal (Lebesgue) measurable controls to reach $x_{f}$ in time $T$.

Remark 4.4: It will become an interesting project to extend the technology in [10] and [3] in order to show the nonexistence of optimal trajectories in an alternative way.

Remark 4.5: For $x_{f}=x_{0}$, the control $u=0$ with $t_{f}=0$ is trivially the time-optimal control.

\section{Pontryagin MaXimum PRinciple}

We derive, from the Pontryagin Maximum Principle (PMP), a necessary condition which time-optimal trajectories must satisfy. Let us define the PMP Hamiltonian $H$ for the time-optimal control as follows (see [12] and [2]):

$$
H\left(x, y, \lambda_{x}, \lambda_{y}, u\right)=\lambda_{x} \alpha u^{2}+\left(\lambda_{x} y+\lambda_{y}\right) u-c y \lambda_{y}
$$

where $\left(\lambda_{x}, \lambda_{y}\right)$ is a covector. Let

$$
M^{\circ}\left(x, y, \lambda_{x}, \lambda_{y}\right)=\max _{|u| \leq 1} H\left(x, y, \lambda_{x}, \lambda_{y}, u\right) .
$$

The application of the PMP in [12] gives:

Theorem 5.1: Consider system (1), (2) with conditions (3)-(6). Let $u(t)$ be a time-optimal control and $(x(t), y(t))$ be the corresponding trajectory. Then, it is necessary that there exists a continuous covector $\left(\lambda_{x}(t), \lambda_{y}(t)\right)$, which is not identically zero, such that

$$
\begin{aligned}
\dot{x} & =y u+\alpha u^{2} \\
\dot{y} & =-c y+u \\
\dot{\lambda}_{x} & =0 \\
\dot{\lambda}_{y} & =c \lambda_{y}-\lambda_{x} u .
\end{aligned}
$$

Additionally, the following must be satisfied:

1. $u(t)=\arg \max _{|v| \leq 1} H\left(x(t), y(t), \lambda_{x}(t), \lambda_{y}(t), v\right)$

2. $M^{\circ}\left(x(t), y(t), \lambda_{x}(t), \lambda_{y}(t)\right)=$ constant $\geq 0$

3. $\lambda_{y}\left(t_{f}\right)=0$ (transversality condition).

The boundary conditions of $(x, y)$ and the signs of parameters are given in (3)-(6). The transversality condition in (40) comes from the free final boundary condition on $y$ in (4).

Remark 5.2: Since $x(t) \in \mathbb{R}$, one can equivalently formulate the previous time-optimal control problem as follows:

$\operatorname{maximize} x(T)$

for $T \geq 0$.

\section{ANALYSIS OF EXTREMALS}

We study the dynamics in (34)-(37). We call the trajectories satisfying the dynamics and all the conditions in Theorem 5.1, extremals.

\section{A. The Necessary Positivity of $\Lambda_{x}$}

By (36), $\lambda_{x}=\lambda_{x}(t)$ is constant in $t$, so there can be the following three cases:

$$
\lambda_{x}=0 \quad \lambda_{x}<0 \quad \lambda_{x}>0 .
$$

We will show that extremals exist only if $\lambda_{x}>0$.

First, we assume $\lambda_{x}=0$. Then (36) and (37) become a linear ordinary differential equation in $\left(\lambda_{x}, \lambda_{y}\right)$ with $\left(\lambda_{x}\left(t_{f}\right), \lambda_{y}\left(t_{f}\right)\right)=(0,0)$. By the uniqueness theorem of solutions of ordinary differential equations [1] or by direct computation, we have $\left(\lambda_{x}(t), \lambda_{y}(t)\right)=0$ for all $t$. Hence, by the PMP, there are no optimal trajectories when $\lambda_{x}=0$.

We now assume $\lambda_{x}<0$. Let

$$
\begin{aligned}
\lambda & =\lambda_{y} / \lambda_{x} \\
\tilde{H}(y, \lambda, u) & =H\left(x, y, \lambda_{x}, \lambda_{y}, u\right) / \lambda_{x} \\
& =\alpha u^{2}+(y+\lambda) u-c y \lambda .
\end{aligned}
$$

Then, (38) implies

$$
u=\arg \min _{|v| \leq 1} \tilde{H}(y, \lambda, v) .
$$

One can compute (recall that $\alpha<0$ )

$$
u(t)=-\operatorname{sign}(y(t)+\lambda(t)) .
$$

With this control, the dynamics of $(y, \lambda)$ can be written as

$$
\left[\begin{array}{l}
\dot{y} \\
\dot{\lambda}
\end{array}\right]=\left[\begin{array}{cc}
-c & 0 \\
0 & c
\end{array}\right]\left[\begin{array}{l}
y \\
\lambda
\end{array}\right]+\left[\begin{array}{c}
-1 \\
1
\end{array}\right] \cdot \operatorname{sign}(y+\lambda) .
$$

Suppose $\lambda(0)>0$. Since $y(0)=0, y(0)+\lambda(0)>0$. One can compute the flow of (42)

$$
y(t)=\frac{1}{c}\left(e^{-c t}-1\right) \quad \lambda(t)=\lambda(0) e^{c t}+\frac{1}{c}\left(e^{c t}-1\right) .
$$

It follows

$$
y(t)+\lambda(t)=\lambda(0) e^{c t}+\frac{2}{c}(\cosh (c t)-1)>0 .
$$

Hence, $u(t)=-\operatorname{sign}(y(t)+\lambda(t))=-1$ for all $t \geq 0$, which implies that $\lambda(t)>0$ for all $t \geq 0$. Hence, the transversality condition (40) cannot be satisfied. One can show in the similar manner that the transversality condition cannot be satisfied 
when $\lambda(0)<0$ or $\lambda(0)=0$. Therefore, there are no optimal trajectories if $\lambda_{x}<0$. Hence, we have proved the following claim.

Claim 6.1: Along every extremal, we have $\lambda_{x}>0$.

\section{B. A Necessary Condition on Parameters for the Existence of Optimal Trajectories}

We derive a necessary condition on the parameters $\alpha$ and $c$ so that optimal trajectories exist. By Claim 6.1, we assume $\lambda_{x}>0$ in the following.

Let

$$
\begin{aligned}
\lambda & =\lambda_{y} / \lambda_{x} \\
\tilde{H}(y, \lambda, u) & =H\left(x, y, \lambda_{x}, \lambda_{y}, u\right) / \lambda_{x} \\
& =\alpha u^{2}+(y+\lambda) u-c y \lambda \\
M(y, \lambda) & =\max _{|v| \leq 1} \tilde{H}(y, \lambda, v)
\end{aligned}
$$

and

$$
\begin{aligned}
R_{n} & =\{(y, \lambda) \mid y+\lambda<2 \alpha\} \\
R_{p} & =\{(y, \lambda) \mid y+\lambda>-2 \alpha\} \\
R_{l} & =\{(y, \lambda)|| y+\lambda \mid \leq-2 \alpha\} .
\end{aligned}
$$

By (38) and (44)

$$
u=\arg \max _{|v| \leq 1} \tilde{H}(y, \lambda, v)
$$

Let us compute $u$ in each region of $R_{n}, R_{p}$, and $R_{l}$ and study the dynamics in each region.

First, we consider the case where $(y, \lambda) \in R_{n}$. Then, $u=$ -1 . The $(y, \lambda)$-dynamics become

$$
\dot{y}=-c y-1 \quad \dot{\lambda}=c \lambda+1
$$

where the equilibrium at $(y, \lambda)=(-1 / c,-1 / c)$ is a saddle.

Second, if $(y, \lambda) \in R_{p}$, then $u=1$. The $(y, \lambda)$-dynamics become

$$
\dot{y}=-c y+1 \quad \dot{\lambda}=c \lambda-1
$$

where the equilibrium at $(y, \lambda)=(1 / c, 1 / c)$ is a saddle.

Finally, if $(y, \lambda) \in R_{l}$, then $u=(y+\lambda) /(-2 \alpha)$. The Hamiltonian $M$ becomes

$$
M(y, \lambda)=\frac{(y+\lambda)^{2}}{-4 \alpha}-c y \lambda
$$

and the $(x, y, \lambda)$-dynamics become

$$
\begin{aligned}
\dot{x} & =\frac{y^{2}-\lambda^{2}}{-4 \alpha} \\
{\left[\begin{array}{c}
\dot{y} \\
\dot{\lambda}
\end{array}\right] } & =A\left[\begin{array}{l}
y \\
\lambda
\end{array}\right]
\end{aligned}
$$

where

$$
A=\left[\begin{array}{cc}
-\left(c+\frac{1}{2 \alpha}\right) & -\frac{1}{2 \alpha} \\
\frac{1}{2 \alpha} & \left(c+\frac{1}{2 \alpha}\right)
\end{array}\right] .
$$

The matrix $A$ satisfies

$$
\operatorname{tr} A=0 \quad \operatorname{det} A=c(1+\alpha c) /(-\alpha) .
$$

The type of the equilibrium at $(y, \lambda)=(0,0)$ depends on the sign of $\operatorname{det} A$.

We now make qualitative phase portraits of the $(y, \lambda)$-dynamics in the following three different cases:

$$
(1+\alpha c)<0 \quad(1+\alpha c)=0 \quad(1+\alpha c)>0 .
$$

Suppose $(1+\alpha c)<0$. Then, $\operatorname{det} A<0$. The origin $(y, \lambda)=$ $(0,0)$ is a saddle point of (49). The (real) eigenvalues of $A$ are given by

$$
\mu_{s}(A)=\frac{\sqrt{\alpha c(1+\alpha c)}}{\alpha} \quad \mu_{u}(A)=\frac{\sqrt{\alpha c(1+\alpha c)}}{-\alpha}
$$

and the corresponding eigenvectors are given by

$$
\begin{aligned}
& v_{s}(A)=\left(1,(\sqrt{-\alpha c}-\sqrt{-(\alpha c+1)})^{2}\right)^{T} \\
& v_{u}(A)=\left(1,(\sqrt{-\alpha c}+\sqrt{-(\alpha c+1)})^{2}\right)^{T} .
\end{aligned}
$$

Notice that $\mu_{s}(A)<0$ and $\mu_{u}(A)>0$. The second (or $\lambda$-) components of $v_{s}(A)$ and $v_{u}(A)$ satisfy

$$
0<v_{s, \lambda}(A)<1<v_{u, \lambda}(A)
$$

because $(1+\alpha c)<0$. In addition, the two saddle points $\pm(1 / c, 1 / c)$ of the dynamics in (45) and (46) do not belong to $R_{n} \cup R_{p}$ but to $R_{l}$ since $(1+\alpha c)<0$. Gathering the information in each region of $R_{n}, R_{p}$, and $R_{l}$, we can draw a phase portrait of the $(y, \lambda)$-dynamics, qualitatively, in Fig. 2. By (51), the slope of the unstable (respectively, stable) manifold of the origin is greater (respectively, smaller) than 1 . As $y(0)=0$ and $\lambda\left(t_{f}\right)=0$, time-optimal trajectories must start from the $\lambda$-axis and ends on the $y$-axis in the $y-\lambda$ plane. However, there are no such orbits in Fig. 2 because the stable and unstable manifolds of the saddle points $\pm(1 / c, 1 / c)$ prevent it in $R_{n} \cup R_{p}$. This implies that there are no optimal trajectories when $\lambda_{x}>0$ and $(1+\alpha c)<0$.

If $(1+\alpha c)=0$, then the matrix $A$ in (50) becomes

$$
A=\left[\begin{array}{ll}
-\frac{c}{2} & \frac{c}{2} \\
-\frac{c}{2} & \frac{c}{2}
\end{array}\right] .
$$

The integration of (49) gives $\lambda(t)=y(t)+c$ where $c$ is a constant. In particular, the line $\lambda=y$ in $R_{l}$ is a set of equilibria. The phase portrait in the $y-\lambda$ plane is given in Fig. 3. One can see that no trajectories starting from the $\lambda$-axis reach the $y$-axis. 


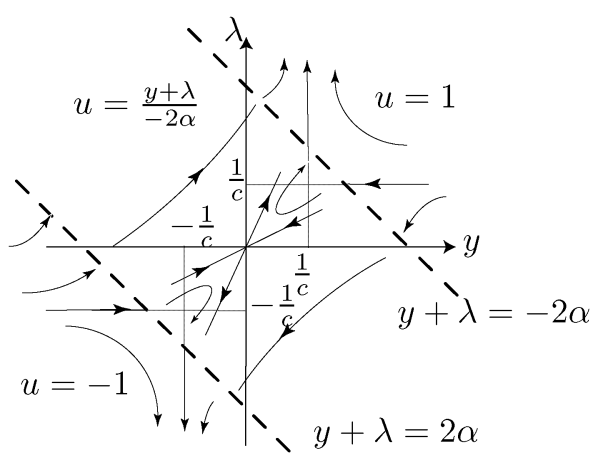

Fig. 2. Phase portrait in the $y-\lambda$ plane when $\lambda_{x}>0$ and $(1+\alpha c)<0$.

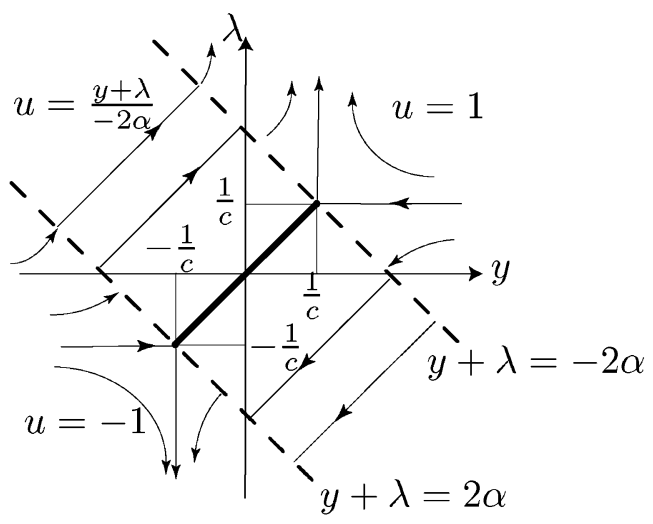

Fig. 3. Phase portrait in the $y-\lambda$ plane when $\lambda_{x}>0$ and $(1+\alpha c)=0$. The segment between $(-1 / c,-1 / c)$ and $(1 / c, 1 / c)$ is the set of equilibria.

Hence, there are no time-optimal trajectories when $\lambda_{x}>0$ and $(1+\alpha c)=0$.

We have, so far, proved the following.

Claim 6.2: Time-optimal trajectories exist only if $(1+\alpha c)>$ 0 .

When $(1+\alpha c)>0$, the fixed point $(y, \lambda)=(0,0)$ of the dynamics (49) is a center as $\operatorname{det} A>0$ with $A$ in (50). In this case, there are two possible qualitatively different phase portraits of the $(y, \lambda)$-dynamics depending on the position of the $\lambda$-intercept of the switching line $y+\lambda=-2 \alpha$ relative to the point $(0,1 / c)$ on the $\lambda$-axis. They are given in Fig. 4 depending on the sign of $(1+2 \alpha c)$, with control

$$
u= \begin{cases}+1, & \text { if } y+\lambda>-2 \alpha \\ \frac{y+\lambda}{-2 \alpha}, & \text { if }|y+\lambda| \leq-2 \alpha \\ -1, & \text { if } y+\lambda<2 \alpha .\end{cases}
$$

We will show that the $(y, \lambda)$-projection of optimal trajectories must be contained in the shaded region in Fig. 4. In the rest of this paper, we assume that $(1+\alpha c)>0$.

\section{Discrete Symmetry}

We will find $\mathbb{Z}_{2} \times \mathbb{Z}_{2}$ symmetry in the dynamics. Define the following maps:

$$
\begin{aligned}
& S_{1}:(x, y, \lambda) \mapsto(x, \lambda, y) \\
& S_{2}:(x, y, \lambda) \mapsto(x,-\lambda,-y) . \\
& S_{3}:=S_{1} \circ S_{2}:(x, y, \lambda) \mapsto(x,-y,-\lambda) .
\end{aligned}
$$

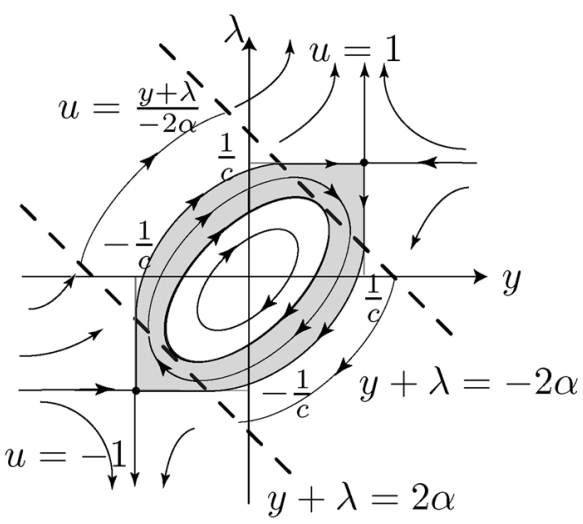

(a)

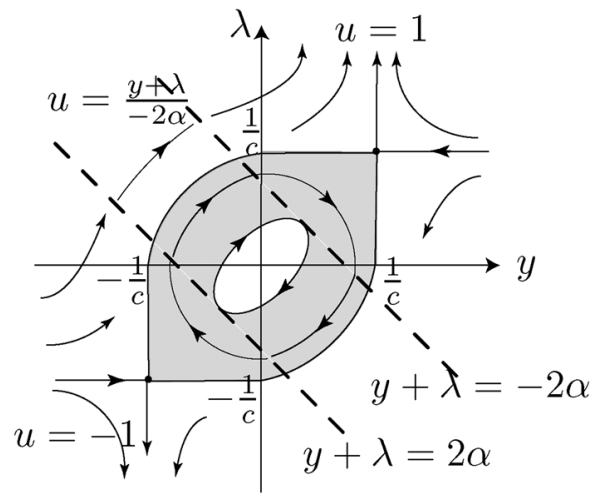

(b)

Fig. 4. Phase portrait in the $y-\lambda$ plane when $\lambda_{x}>0$ and $(1+\alpha c)>0$. Depending on the sign of $(1+2 \alpha c)$, the $\lambda$-intercept, $-2 \alpha$, of the switching line $y+\lambda=-2 \alpha$ is greater or less than $1 / c$. (a) $(1+2 \alpha c) \leq 0$. (b) $(1+2 \alpha c)>0$.

Denoting by $Z_{l}(x, y, \lambda)$ the vector field in (7)-(9) with the linear control $u=(y+\lambda) /(-2 \alpha)$ on $\mathbb{R} \times R_{l}$, i.e., that in (48) and (49), we obtain

$$
S_{i} \circ Z_{l}=-Z_{l} \circ S_{i}, \quad i=1,2 .
$$

The linear vector field $Z_{l}$ is invariant under the reflections, $S_{1}$ and $S_{2}$, up to the time-reversal, and it is invariant under the reflection $S_{3}$ without time-reversal. The region $\mathbb{R} \times R_{l}$ is invariant under $Z_{i}, i=1,2$. Notice this symmetry in the phase portraits in region $R_{l}$ of Fig. 4. This symmetry gives us useful information as follows. Consider a trajectory of $Z_{l}$ whose $(y, \lambda)$-projected image is contained in $R_{l}$ as in Fig. 5. The duration $\Delta t_{A B}$ from $A$ to $B$ along the trajectory with $u=(y+\lambda) /(-2 \alpha)$ in the $y-\lambda$ plane is the same as $\Delta t_{S_{i}(B) S_{i}(A)}, i=1,2$. Also, the corresponding (positive or negative) increments in $x$ satisfy

$$
\Delta x_{A B}=-\Delta x_{S_{i}(B) S_{i}(A)}, \quad i=1,2 .
$$

This implies that there cannot be any optimal trajectories in the white region surrounded by the shaded region in Fig. 4 because an arbitrary trajectory starting from the $\lambda$-axis and ending at the $y$-axis can be decomposed into parts, each of which is invariant under $S_{1}$ or $S_{2}$, so $\Delta x=0$ along the whole trajectory. However, the time-optimal control for $\Delta x=0$ is $u=0$ with 


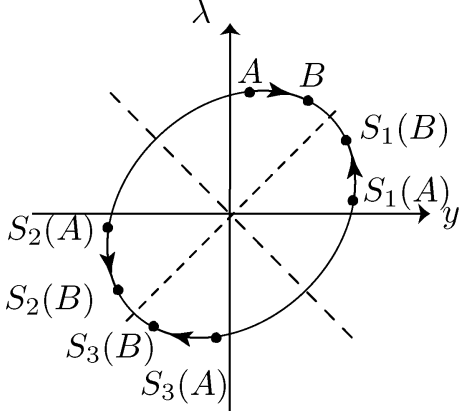

Fig. 5. $\mathbb{Z}_{2} \times \mathbb{Z}_{2}$-symmetry in the linear region.

$t_{f}=0$; see Remark 4.5. Therefore, all time-optimal trajectories are contained in the shaded regions in Fig. 4 because only the trajectories in the shaded region can satisfy $y(0)=0$ and $\lambda\left(t_{f}\right)=0$.

Let us consider the symmetry $S_{3}$ in (55). Let $Z$ be the vector field in (7)-(9) on the whole domain, $\mathbb{R}^{3}=\mathbb{R} \times\left(R_{n} \cup R_{p} \cup R_{l}\right)$. One can check

$$
\begin{aligned}
S_{3}\left(\mathbb{R} \times R_{n}\right) & =\mathbb{R} \times R_{p} \\
S_{3}\left(\mathbb{R} \times R_{l}\right) & =\mathbb{R} \times R_{l} \\
S_{3} \circ Z & =Z \circ S_{3} \\
\Delta t_{A B} & =\Delta t_{S_{3}(A) S_{3}(B)} .
\end{aligned}
$$

Hence, for example

$$
\Delta x_{A B}=\Delta x_{S_{3}(A) S_{3}(B)}
$$

in Fig. 5.

\section{Definition, Monotonicity, and Positivity of $X_{1}$}

Let us first define the basic region in the $y-\lambda$ plane. In each phase portrait in Fig. 4 , we denote by $\mathcal{A}$ the interior of the shaded region. The basic region $\mathcal{B}$ is defined by

$$
\mathcal{B}=\left\{(y, \lambda) \in \mathcal{A} \subset \mathbb{R}^{2} \mid y \geq 0, \lambda \geq 0\right\} .
$$

See Fig. 6 for an illustration of the basic region.

Given an extremal $\gamma(t)=(x(t), y(t), \lambda(t)), 0 \leq t \leq T$, the arc $\gamma\left(\left[t_{1}, t_{2}\right]\right)$ with $0 \leq t_{1}<t_{2} \leq T$ is called a basic arc of $\gamma$ if $y\left(t_{1}\right)=0, \lambda\left(t_{2}\right)=0$ and

$$
\tilde{\pi}\left(\gamma\left(\left[t_{1}, t_{2}\right]\right)\right) \subset \mathcal{B} \cup \tilde{S}_{3}(\mathcal{B})
$$

where $\tilde{\pi}(x, y, \lambda)=(y, \lambda)$ and $\tilde{S}_{3}(y, \lambda)=(-y,-\lambda)$.

We denote by $\Lambda$ the open interval which is the intersection of the positive $\lambda$-axis with the basic region; see Fig. 6 . It is given in (13) and (14), whose derivation will be made later. Let us construct a function $X_{1}$ of $\lambda_{0} \in \Lambda$, which measures the (signed) increment of $x$ along a basic arc starting with $\lambda(0)=\lambda_{0}$. The

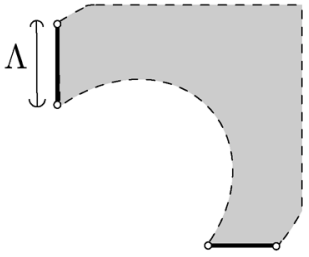

(a)

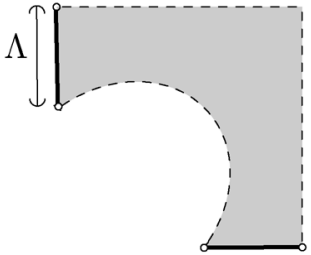

(b)
Fig. 6. Basic region and the domain $\Lambda$ of the map $X_{1}$. (a) $(1+2 \alpha c) \leq 0$ (b) $(1+2 \alpha c)>0$.

construction of $X_{1}$ and $\Lambda$ is made in the following two separate cases:

$$
(1+2 \alpha c) \leq 0 \quad(1+2 \alpha c)>0 .
$$

Case $(1+2 \alpha c) \leq 0$ : The domain $\Lambda$ of $X_{1}$ is computed as follows:

$$
\begin{aligned}
\Lambda= & \left\{\lambda \in \mathbb{R}_{+} \mid M(-\alpha,-\alpha)<M(0, \lambda)\right. \\
& <M(-(1+2 \alpha c) / c, 1 / c)\} \\
= & (-2 \alpha \sqrt{1+\alpha c},-2 \alpha \sqrt{(1+\alpha c) /(-\alpha c)})
\end{aligned}
$$

which is exactly (13), where the Hamiltonian $M$ is given in (47). The switching line $\{y+\lambda=-2 \alpha\}$ is tangent to the level set $\{M(y, \lambda)=M(-\alpha,-\alpha)\}$ at $(-\alpha,-\alpha)$. The level set $\{M(y, \lambda)=M(-(1+2 \alpha c) / c, 1 / c)\}$ goes through the intersection of the switching line $\{y+\lambda=-2 \alpha\}$ and a stable manifold $\{\lambda=1 / c\}$ of $(1 / c, 1 / c)$; see Fig. 4(a). Notice in Fig. 4(a) that all the basic arcs are of form $\Gamma_{+}^{\lambda_{0}}$ defined in (17). Namely, basic arcs in the basic region are like arc $A B C D$ in Fig. 7(a). Let $A=\left(0, \lambda_{0}\right)$ in Fig. 7(a). Then, $D=\left(\lambda_{0}, 0\right)$ by the $S_{1}$ symmetry. The two points $B$ and $C$ in Fig. 7(a) are the intersection of the level set of $M$

$$
D_{1}=\left\{(y, \lambda) \mid M(y, \lambda)=M\left(0, \lambda_{0}\right)\right\}
$$

and the switching line

$$
D_{2}=\{(y, \lambda) \mid y+\lambda=-2 \alpha\} \text {. }
$$

These points are symmetric to each other with respect to $S_{1}$ in (53) because $D_{1}$ and $D_{2}$ are invariant under $S_{1}$. The $y$-coordinates of $B$ and $C$ are given by

$$
\begin{aligned}
& y_{B}\left(\lambda_{0}\right)=-\alpha-\sqrt{\alpha^{2}+\left(\lambda_{0}^{2}-4 \alpha^{2}\right) /(-4 \alpha c)} \\
& y_{C}\left(\lambda_{0}\right)=-y_{B}\left(\lambda_{0}\right)-2 \alpha .
\end{aligned}
$$

Due to the $S_{1}$ symmetry, the increments in $x$ along $A B$ and $C D$ cancel each other, i.e.,

$$
\Delta x_{A B}+\Delta x_{C D}=0 .
$$




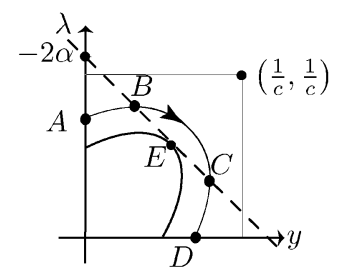

(a)

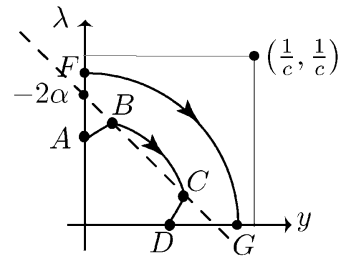

(b)
Fig. 7. Construction of the $x$-increment map $X_{1}$. (a) $(1+2 \alpha c) \leq 0$. (b) $(1+$ $2 \alpha c)>0$.

\section{Hence}

$$
\begin{aligned}
X_{1}\left(\lambda_{0}\right)= & \Delta x_{B C}=\int_{y_{B}\left(\lambda_{0}\right)}^{y_{C}\left(\lambda_{0}\right)} \frac{\dot{x}}{\dot{y}} d y \\
= & \int_{y_{B}}^{y_{C}} \frac{y+\alpha}{-c y+1} d y \\
= & \frac{1}{c}\left[-2 \alpha-2 y_{C}\left(\lambda_{0}\right)\right. \\
& \left.\quad+\left(\alpha+\frac{1}{c}\right) \ln \left(\frac{1+2 \alpha c+c y_{C}\left(\lambda_{0}\right)}{1-c y_{C}\left(\lambda_{0}\right)}\right)\right]
\end{aligned}
$$

with $y_{C}\left(\lambda_{0}\right)$ in (61). Regarding $X_{1}$ as a function of $y_{C}$, one can see

$$
\frac{d X_{1}}{d y_{C}}=\frac{2\left(y_{C}+\alpha\right)^{2}}{c\left(\frac{1}{c}+2 \alpha+y_{C}\right)\left(\frac{1}{c}-y_{C}\right)} \geq 0
$$

on $y_{C}(\Lambda)=(-\alpha, 1 / c)$ because $(1+\alpha c)>0$. Since $y_{C}$ in (61) is a strictly increasing function of $\lambda_{0}$, it follows that $X_{1}$ is a strictly increasing function on $\Lambda$. Note that

$$
\begin{gathered}
\lim _{\lambda_{0} \rightarrow-2 \alpha \sqrt{1+\alpha c}} X_{1}\left(\lambda_{0}\right)=\lim _{y_{C} \rightarrow-\alpha} X_{1}\left(y_{C}\right)=0 \\
\lim _{\lambda_{0} \rightarrow-2 \alpha \sqrt{\frac{1+\alpha c}{-\alpha c}}} X_{1}\left(\lambda_{0}\right)=\lim _{y_{C} \rightarrow 1 / c} X_{1}\left(y_{C}\right)=+\infty
\end{gathered}
$$

It follows that $X_{1}(\Lambda)=(0, \infty)$.

Case $(1+2 \alpha c)>0$ : The domain $\Lambda$ of $X_{1}$ is given by

$$
\Lambda=\Lambda_{1} \cup \Lambda_{2}=(-2 \alpha \sqrt{1+\alpha c},-2 \alpha) \cup[-2 \alpha, 1 / c)
$$

where the left end $-2 \alpha \sqrt{1+\alpha c}$ of $\Lambda$ is computed from $M(0, \lambda)=M(-\alpha,-\alpha)$ and the right end $1 / c$ is just the intersection of the stable manifold of the fixed point $(1 / c, 1 / c)$, with the $\lambda$-axis in Fig. 4(b). As $-2 \alpha \sqrt{1+\alpha c}<-2 \alpha<1 / c$, the decomposition of $\Lambda$ into $\Lambda_{1}$ and $\Lambda_{2}$ is valid. Notice in Fig. 4(b) that there are two different kinds of basic arcs in the basic region. If $\lambda_{0} \in \Lambda_{1}$, then the trajectory is like $A B C D$ in Fig. 7(b), i.e., $\Gamma_{+}^{\lambda_{0}}$ in (17). In this case, the control is given by $u=(y+\lambda) /(-2 \alpha)$ on $A B$ and $C D$ and $u=+1$ on $B C$. If $\lambda_{0} \in \Lambda_{2}$, then the trajectory is like $F G$ where the control is given by $u=+1$.

First, we consider the case where $\lambda_{0} \in \Lambda_{1}$. Let $y_{B}$ and $y_{C}$ be the $y$-coordinates of the intersections points of $D_{1}$ and $D_{2}$ in
(58) and (59). The formulas of $y_{B}$ and $y_{C}$ are given in (60) and (61). Due to the $S_{1}$ symmetry, relation (62) holds. Hence

$$
\begin{aligned}
& X_{1}\left(\lambda_{0}\right)=\frac{1}{c}[-2 \alpha-2 y_{C}\left(\lambda_{0}\right) \\
&\left.+\left(\alpha+\frac{1}{c}\right) \ln \left(\frac{1+2 \alpha c+c y_{C}\left(\lambda_{0}\right)}{1-c y_{C}\left(\lambda_{0}\right)}\right)\right]
\end{aligned}
$$

which coincides with (63). One can check that (64) is still valid on $y_{C}\left(\Lambda_{1}\right)$, and that $y_{C}$ is a strictly increasing function of $\lambda_{0}$ on $\Lambda_{1}$. Hence, $X_{1}$ is a strictly increasing function of $\lambda_{0}$ on $\Lambda_{1}$.

We now consider the case that $\lambda_{0} \in \Lambda_{2}$. We have

$$
\begin{aligned}
X_{1}\left(\lambda_{0}\right) & =\int_{y_{F}}^{y_{G}} \frac{\dot{x}}{\dot{y}} d y=\int_{0}^{\lambda_{0}} \frac{y+\alpha}{-c y+1} d y \\
& =\frac{1}{c}\left[-\lambda_{0}+\left(\alpha+\frac{1}{c}\right) \ln \left(\frac{1}{1-c \lambda_{0}}\right)\right] .
\end{aligned}
$$

Notice the continuity of $X_{1}$ at $\lambda_{0}=-2 \alpha$ from (63) and (67). Since

$$
\frac{d X_{1}}{d \lambda_{0}}=\frac{\lambda_{0}+\alpha}{1-c \lambda_{0}}>0
$$

for $\lambda_{0} \in \Lambda_{2}$, the function $X_{1}\left(\lambda_{0}\right)$ is strictly increasing on $\Lambda_{2}$. Notice that

$$
\lim _{\lambda_{0} \rightarrow 1 / c} X_{1}\left(\lambda_{0}\right)=+\infty
$$

From (65) and (68), it follows that $X_{1}(\Lambda)=(0, \infty)$. We conclude that the map $X_{1}\left(\lambda_{0}\right)$ is strictly increasing on $\Lambda$ and its image is $(0, \infty)$.

We have proved the following.

Claim 6.3: Irrespective of the sign of $(1+2 \alpha c)$, the map $X_{1}\left(\lambda_{0}\right)$ on $\Lambda$, which is the displacement in $x$ along the basic arc with $\lambda(0)=\lambda_{0}$ in the basic region, is a strictly increasing function and its range is $(0, \infty)$.

\section{E. Duration of Basic Arcs and Idling Arcs}

We compute the duration of basic arcs. Given an extremal $(x(t), y(t), \lambda(t))$ with $(y(0), \lambda(0))=\left(0, \lambda_{0}\right) \in 0 \times \Lambda$, let $T_{1}$ be the smallest $t>0$ such that $(y(t), \lambda(t))=\left(\lambda_{0}, 0\right)$ where $\Lambda$ is defined in (13) and (14). We can regard $T_{1}$ as a function of $\lambda_{0} \in \Lambda$. Recall the three logic sentences, $P_{1}, P_{2}$ and $Q$ defined in Section II. We will compute $T_{1}$ separately for the following two cases:

$$
[\mathrm{P} 1 \vee \mathrm{Q}] \text { and } \mathrm{P} 2 .
$$

We begin with the case of $[\mathrm{P} 1 \quad \vee \quad \mathrm{Q}]$. The $(y, \lambda)$-projection of a basic arc is like the arc $A B C D$ in Fig. 7(a) and (b). It consists of the three arcs, $A B, B C$, and $C D$ where $u=(y+$ $\lambda) /(-2 \alpha)$ on $A B$ and $C D$ and $u=+1$ on $C D$. Let us compute the flight time $\Delta t_{A B}$ from $A$ to $B$. By the $S_{1}$ symmetry, the flight time $\Delta t_{C D}$ is the same as $\Delta t_{A B}$. The dynamics are given 
in (48)-(50) with the initial condition $(y(0), \lambda(0))=\left(0, \lambda_{0}\right)$. The solution is given by

$$
\left\{\begin{array}{l}
y(t)=\frac{\lambda_{0}}{-2 \alpha \omega} \sin (\omega t) \\
\lambda(t)=\lambda_{0} \cos (\omega t)+\frac{\lambda_{0}(1+2 \alpha c)}{2 \alpha \omega} \sin (\omega t) .
\end{array}\right.
$$

where

$$
\omega=\sqrt{\operatorname{det} A}=\sqrt{c(1+\alpha c) /(-\alpha)}>0
$$

Thus

$$
\Delta t_{A B}\left(\lambda_{0}\right)=\Delta t_{C D}\left(\lambda_{0}\right)=\frac{1}{\omega} \sin ^{-1}\left(\frac{-2 \alpha \omega y_{B}\left(\lambda_{0}\right)}{\lambda_{0}}\right)
$$

where $y_{B}\left(\lambda_{0}\right)$ is given in (60). We now compute the flight time $\Delta t_{B C}$ for $B C$. For this purpose, the corresponding $y$-dynamics can be written as

$$
\dot{y}=-c y+1 \quad y(0)=y_{B} \quad y\left(\Delta t_{B C}\right)=y_{C}
$$

where $y_{B}$ and $y_{C}$ are given in (60) and (61). Direct integration yields

$$
\Delta t_{B C}\left(\lambda_{0}\right)=\frac{1}{c} \ln \left(\frac{1-c y_{B}\left(\lambda_{0}\right)}{1-c y_{C}\left(\lambda_{0}\right)}\right) .
$$

By (71) and (72), the total flight time $T_{1}$ of $A B C D$ is given by

$$
\begin{array}{r}
T_{1}\left(\lambda_{0}\right)=\frac{2}{\omega} \sin ^{-1}\left(\frac{-2 \alpha \omega y_{B}\left(\lambda_{0}\right)}{\lambda_{0}}\right) \\
+\frac{1}{c} \ln \left(\frac{1-c y_{B}\left(\lambda_{0}\right)}{1-c y_{C}\left(\lambda_{0}\right)}\right) .
\end{array}
$$

We now consider the case of $\mathrm{P} 2$. The $y$-dynamics is

$$
\dot{y}=-c y+1 \quad y(0)=0 \quad y\left(T_{1}\right)=\lambda_{0} .
$$

Direct integration yields

$$
T_{1}\left(\lambda_{0}\right)=\frac{1}{c} \ln \left(\frac{1}{1-c \lambda_{0}}\right) .
$$

We have so far verified the formula of $T_{1}$ in (16).

We make a remark on the relation between $X_{1}$ and $T_{1}$. Recall that $X_{1}\left(\lambda_{0}\right)$ is a bijection from $\Lambda$ to $(0, \infty)$. The corresponding flight time $T_{1}\left(\lambda_{0}\right)$ can be regarded as function of $X_{1} \in(0, \infty)$ as follows:

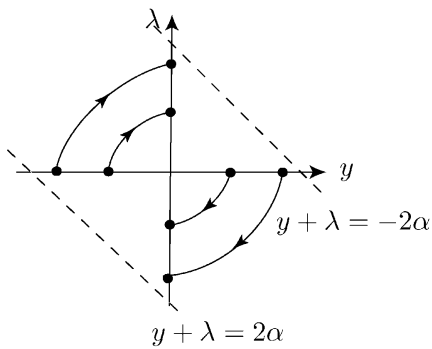

Fig. 8. Idling arcs do not contribute to the net displacement of a particle.

Recall the definition of idling arcs in Section II. An idling arc is an arc whose $(y, \lambda)$-projection starts from the positive (respectively, negative) $y$-axis, goes through the fourth quadrant (respectively, the second quadrant) in the region of $R_{l}$, and ends at the negative (respectively, positive) $\lambda$-axis; see Fig. 8 . By the $S_{2}$ symmetry, they do not contribute to the displacement of $x$. Idling arcs occur in the case of [P1 $\vee \mathrm{Q}]$. The flight time, $T_{\text {idling}}$, which we call idling time, of idling arcs is given by

$$
T_{\text {idling }}=\frac{1}{\omega} \sin ^{-1}(-2 \alpha \omega)
$$

with $\omega$ in (70). Notice that the idling time is independent of the coordinates of the initial point on the $y$-axis.

\section{F. Construction and Uniqueness of Optimal Trajectories}

We investigate the issue of the construction and uniqueness of the time-optimal trajectory. Recall that the $(y, \lambda)$ projection of optimal trajectories must start from the $\lambda$-axis and end at the $y$-axis as $y(0)=0$ and $\lambda\left(t_{f}\right)=0$. Because of the $S_{3}$ symmetry, if a trajectory with $\lambda(0) \in \Lambda$ is optimal, then its $S_{3}$ image is also optimal. Hence, without loss of generality, we will always give proofs only for optimal trajectories starting with $\lambda(0) \in \Lambda$.

We call an extremal an $n$-shot extremal where $n \in \mathbb{N}$ if the maximum number of basic arcs in the extremal is $n$. Equivalently, we say that an extremal is an $n$-shot extremal if its $(y, \lambda)$ projection meets with the $y$-axis $n$ times. For example, the extremal whose $(y, \lambda)$ projection is in Fig. 9(a), is a one-shot extremal and that in Fig. 9(b) is a two-shot extremal. The one in Fig. 9(c) corresponds to a multishot extremal. Notice that the $(y, \lambda)$ projection of an $n$-shot extremal with $n \geq 3$ is a closed curve by symmetry. By the discussion in Sections VI-C and VI-D, we know that for a given $x_{f}>x_{0}$ there always exists a unique $n$-shot extremal for each $n \in \mathbb{N}$ with $\lambda(0)=\lambda_{0} \in \Lambda$ reaching $x_{f}$. Hence, we need to know how to find time-optimal ones among them. We divide our discussion into the following two cases.

$$
(3+4 \alpha c) \geq 0 \quad(3+4 \alpha c)<0 .
$$

Case $(3+4 \alpha c) \geq 0$ : We will show that the two one-shot extremals are the time-optimal ones for a given $x_{f}>x_{0}$. Recall
$T_{1}\left(X_{1}\right)=T_{1} \circ \lambda_{0}\left(X_{1}\right)$. 


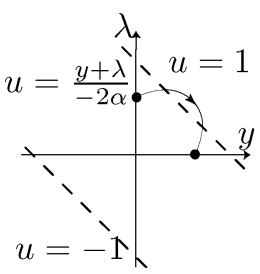

(a)

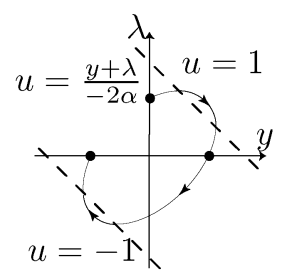

(b)

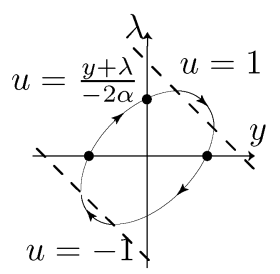

(c)
Fig. 9. There are several types of extremals. Their $(y, \lambda)$-projection must start from the $\lambda$-axis and end on the $y$-axis because of the boundary condition. (a) One-shot extremal, (b) two-shot extremal, and (c) multishot extremal.

the three logic sentences, P1, P2, and R defined in Section II. It is easy to verify

$$
\mathrm{P} 1 \vee \mathrm{P} 2 \Rightarrow \mathrm{R} \text {. }
$$

We will divide our discussion into the following three cases:

$$
\mathrm{P} 2, \mathrm{P} 1,[\mathrm{R} \wedge \neg(\mathrm{P} 1 \vee \mathrm{P} 2)]
$$

We first consider the case of P2. Suppose that the two-shot extremal, FGHIJK, in Fig. 10(a) is time-optimal with a final time $t_{f}>0$ and a control $u$ for $x_{f}>x_{0}$. Let us consider another control $v(t)=1$ for $t \in\left[0, t_{f}\right]$. Let $F G H J^{\prime} K^{\prime}$ be the trajectory due to $v$. By the $S_{2}$ symmetry, $\Delta x_{H I}=0$. Hence, the increment in $x$ due to the control $u$ is given by

$$
\Delta x_{F G H I J K}(u)=\Delta x_{F G H}(u=1)+\Delta x_{S_{3}(I) F G}(u=1)
$$

by the $S_{3}$ symmetry where $S_{3}(I J K)=S_{3}(I) F G$. The increment in $x$ due to the control $v$ is given by

$$
\Delta x_{F G H J^{\prime} K^{\prime}}(v)=\Delta x_{F G H}(v=1)+\Delta x_{H J^{\prime} K^{\prime}}(v=1) .
$$

Along $S_{3}(I) F G H J^{\prime} K^{\prime}, \dot{y}=-c y+1>0$. Let $J^{\prime}$ be the point such that the flight time on $S_{3}(I) F G$ is the same as that on $H J^{\prime}$. Namely, $\Delta t_{S_{3}(I) F G}=\Delta t_{H J^{\prime}}$. Then the flight time on $H I$ is the same as that on $J^{\prime} K^{\prime}$. Notice that

$$
\begin{aligned}
& \Delta x_{S_{3}(I) F G}(u=1) \\
& \quad=\int_{0}^{\Delta t_{S_{3}(I) F G}}\left(y_{1}(t)+\alpha\right) d t \\
& <\int_{0}^{\Delta t_{H J^{\prime}}}\left(y_{2}(t)+\alpha\right) d t \\
& =\Delta x_{H J^{\prime}}(v=1)
\end{aligned}
$$

where $y_{1}(t)=y_{S_{3}(I)} e^{-c t}+(1) /(c)\left(1-e^{-c t}\right), y_{2}(t)=$ $y_{H} e^{-c t}+(1) /(c)\left(1-e^{-c t}\right)$, and $y_{S_{3}(I)}<y_{H}$. On $J^{\prime} K^{\prime}, \dot{x}=y+\alpha>0$ because $y_{H} \geq y_{G}=\lambda_{0} \geq-2 \alpha$ and $\dot{y}>0$ on $J^{\prime} K^{\prime}$. Thus, $\Delta x_{J^{\prime} K^{\prime}}(v=1)>0$. Therefore, $\Delta x_{F G H J^{\prime} K^{\prime}}(v)>\Delta x_{F G H I J K}(u)$. This implies, by the continuity of $t \mapsto x(t) \in \mathbb{R}$, that the trajectory due to the control

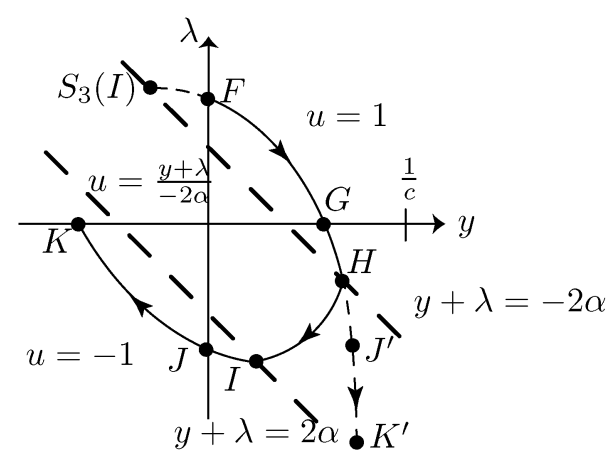

(a)

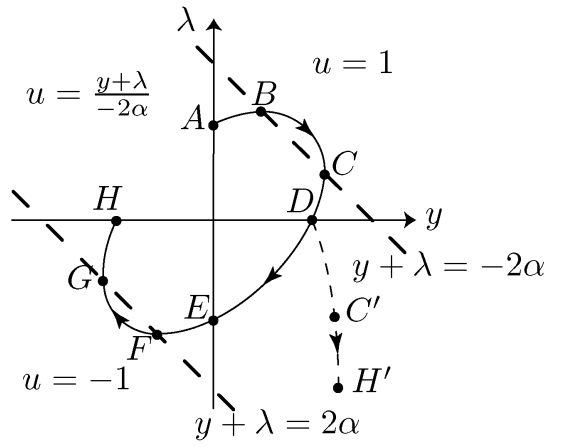

(b)

Fig. 10. One-shot extremal is the optimal one among extremals for a given $x_{f}>x_{0}$ when $(1+2 \alpha c)>0$. (a) $\lambda_{0} \in \Lambda_{2}=[-2 \alpha, 1 / c)$. (b) $\lambda_{0} \in \Lambda_{1}=$ $(-2 \alpha \sqrt{1+\alpha c},-2 \alpha)$.

$v$ reaches $x_{f}$ before $t=t_{f}$, which contradicts the time-optimality of the control $u$. Hence, no two-shot extremals can be time-optimal in the case of P2. Similarly, one can show that no multi-shot extremals can be time-optimal in the case of P2.

We now consider the case of P1. Suppose that a two-shot extremal with $\lambda_{0} \in \Lambda_{1}$ is time-optimal with $t_{f}>0$ and control $u$ for $x_{f}>x_{0}$. Let $A B C D E F G H$ be the trajectory associated with $u$ in Fig. 10(b). Let $t_{1}$ is the time when the trajectory reaches $D$. Let us construct another control $v$ as follows:

$$
v(t)= \begin{cases}u(t), & \text { if } t \in\left[0, t_{1}\right] \\ 1, & \text { if } t \in\left[t_{1}, t_{f}\right] .\end{cases}
$$

Let $A B C D C^{\prime} H^{\prime}$ be the trajectory associated with the control $v$ where $D C^{\prime} H^{\prime}$ corresponds to the time interval $\left[t_{1}, t_{f}\right]$, and we choose $C^{\prime}$ such that $\Delta t_{B C}=\Delta t_{D C^{\prime}}$. By the $S_{1}$ and $S_{2}$ symmetry

$$
\Delta x_{A B}+\Delta x_{C D}=\Delta x_{E F}+\Delta x_{G H}=0 .
$$

The total increment in $x$ due to the control $u$ is given by

$$
\Delta x(u)=2 \Delta x_{B C} .
$$

The total increment in $x$ due to the control $v$ is

$$
\Delta x(v)=\Delta x_{B C}+\Delta x_{D C^{\prime}}+\Delta x_{C^{\prime} H^{\prime}}
$$


By the $S_{1}$ symmetry, $y_{D}=\lambda_{0}$, so

$$
y_{D}=\lambda_{0}>-2 \alpha \sqrt{1+\alpha c} \geq-\alpha \geq y_{B}
$$

where $\lambda_{0} \in \Lambda,(3+4 \alpha c) \geq 0$ and (60) were used for the three inequalities. As $\dot{y}=-c y+1>0$ on $B C$ and $D C^{\prime}$, we have

$$
\begin{aligned}
\Delta x_{B C} & =\int_{0}^{\Delta t_{B C}}\left(y_{1}(t)+\alpha\right) d t \\
& <\int_{0}^{\Delta t_{D C^{\prime}}}\left(y_{2}(t)+\alpha\right) d t=\Delta x_{D C^{\prime}}
\end{aligned}
$$

where $y_{1}(t)=y_{B} e^{-c t}+(1) /(c)\left(1-e^{-c t}\right)$ and

$$
y_{2}(t)=y_{D} e^{-c t}+\frac{1}{c}\left(1-e^{-c t}\right) .
$$

It is straightforward to see

$$
\Delta x_{C^{\prime} H^{\prime}}>0 .
$$

By (77), (78), (80), and (81),

$$
\Delta x(v)>\Delta x(u) .
$$

This implies that the trajectory due to the control $v$ reaches $x_{f}$ before $t=t_{f}$, which contradicts the time-optimality of $u$. Therefore, there are no two-shot time-optimal trajectories in the case of P1. In the similar manner, one can show that no multishot extremals can be time-optimal in the case of P1.

We now consider the case of $[\mathrm{R} \wedge \neg(\mathrm{P} 1 \vee \mathrm{P} 2)]$. In this case, all the basic arcs are of form $\Gamma_{ \pm}^{\lambda_{0}}$ in (17). Notice that for the case of P1 we only used the fact that the basic arcs are of form $\Gamma_{ \pm}^{\lambda_{0}}$ and $(3+4 \alpha c) \geq 0$ where the latter was used in (79). Hence, the case of $[\mathrm{R} \wedge \neg(\mathrm{P} 1 \vee \mathrm{P} 2)]$ can be handled in the same way as the case of $\mathrm{P} 1$, to conclude that no multishot extremals are time-optimal.

So far, we have showed that multishot extremals cannot be time-optimal when $(3+4 \alpha c) \geq 0$. By the discussion in Section VI-D, we know that there exists a unique one-shot extremal with $\lambda_{0} \in \Lambda$, or a basic arc, for a given $x_{f}>x_{0}$ such that $x\left(t_{f}\right)=x_{f}$. Hence, this basic arc and its image under the reflection $S_{3}$ are the only optimal trajectories.

Claim 6.4: If $(3+4 \alpha c) \geq 0$, then there are exactly two time optimal trajectories for $x_{f}>x_{0}$. One is the one-shot extremal $\Gamma_{+}^{\lambda_{0}}$ (or, the basic arc) with $\lambda(0)=\lambda_{0}=X_{1}^{-1}\left(x_{f}-x_{0}\right)$ and the other is its image by the reflection $S_{3}$ (see also Section II). The corresponding minimum time is $T_{1}\left(\lambda_{0}\right)$. The maps $X_{1}$ and $T_{1}$ are defined in (15) and (16).

Case $(3+4 \alpha c)<0$ : In this case, unlike the case of $(3+$ $4 \alpha c) \geq 0$, we have no general proof that only one-shot extremals are time-optimal. Instead we provide a finite algorithm of finding all time-optimal trajectories for $x_{f}>x_{0}$.

Take $x_{f}>x_{0}$. For each $n \in \mathbb{N}$, there exists a unique $n$-shot extremal with $\lambda(0)=\lambda_{0, n}:=X_{1}^{-1}\left(\left(x_{f}-x_{0}\right) / n\right)$ reaching $x_{f}$. Since $(3+4 \alpha c)<0$ implies $(1+2 \alpha c) \leq 0$, this $n$-shot extremal consists of $n$ one-shot extremals and $(n-1)$ idling arcs between the $n$ one-shot extremals; see (19), Figs. 7(a), and 9(c).
Each of the $n$ one-shot extremals contributes $\left(x_{f}-x_{0}\right) / n$ to the increment in $x$, and each idling arc makes zero contribution. By this decomposition, the total time cost $T_{n}$ for this $n$-shot extremal is

$$
T_{n}=n \cdot T_{1} \circ X_{1}^{-1}\left(\left(x_{f}-x_{0}\right) / n\right)+(n-1) \cdot T_{\text {idling }}
$$

where $T_{1} \circ X_{1}^{-1}\left(\left(x_{f}-x_{0}\right) / n\right)$ is the flight time corresponding to the increment $\left(x_{f}-x_{0}\right) / n$ in $x$ where $X_{1}, T_{1}$, and $T_{\text {idling }}$ are given in (63), (73), and (76). There exists $N \in \mathbb{N}$ such that

$$
T_{1}=T_{1} \circ X_{1}^{-1}\left(x_{f}-x_{0}\right)<N \cdot T_{\text {idling }}
$$

which implies

$$
T_{1}<T_{n} \quad \forall n \geq(N+1) .
$$

Thus, all possible time-optimal trajectories are among the first $N$ extremals. Choose $k \in\{1, \ldots, N\}$ such that $T_{k}=\min \left\{T_{i}\right.$ : $1 \leq i \leq N\}$. Then, the $k$-shot extremal is a time-optimal trajectory. Such $k$ 's give all the time-optimal trajectories corresponding to $x_{f}>x_{0}$. This proves the procedure [B.1]-[B.4] in Section II, which can be summarized as follows.

Claim 6.5: If $(3+4 \alpha c)<0$, then there is a finite and explicit procedure ([B.1]-[B.4] in Section II) of finding all time-optimal trajectories and the corresponding minimum time for $x_{f}>x_{0}$.

As a remark, we give a practical way of showing that the oneshot extremals are the unique time-optimal trajectories. First, with (63) and (73), one draws the graph $\left(X_{1}, T_{1}\left(X_{1}\right)\right)$ in (75). Suppose that it is strictly concave. Then

$T_{1}=T_{1}\left(X_{1}=\Delta x\right)<n \cdot T_{1}\left(X_{1}=\Delta x / n\right) \quad \forall n=2,3, \ldots$ which, with (82), implies that $T_{1}<T_{n}$ for all $n \geq 2$. Hence, the two one-shot extremals are the only time-optimal trajectories if $T_{1}\left(X_{1}\right)$ is a strictly concave function of $X_{1}$.

\section{G. Initial Undershoots}

One can check that for $\lambda(0) \in \Lambda$, every extremal has $\dot{x}(t) \leq 0$ from $t=0$ until $y(t)$, which is initially zero, becomes $-\alpha>0$. In other words, $x(t)$ goes through an initial undershoot. We will compute this undershoot and its duration. Here, we do not give detailed computation because the methodology is very similar to those in Sections VI-D and VI-E. Recall the three logic sentences, P1, P2, and Q defined in Section II.

Let us first consider the case of $[\mathrm{P} 1 \vee \mathrm{Q}]$. The undershoot consists of two parts in Fig. 7(a) and (b): $A B$ and $B \rightarrow(-\alpha, \bar{\lambda})$ for some $\bar{\lambda}$ where $(-\alpha, \bar{\lambda})$ lies between $B$ and $C$ on the trajectory. One can compute the duration $T_{\text {under }}\left(\lambda_{0}\right)$ of the undershoot as

$$
T_{\text {under }}\left(\lambda_{0}\right)=\Delta t_{A B}\left(\lambda_{0}\right)+\frac{1}{c} \ln \left(\frac{1-c y_{B}\left(\lambda_{0}\right)}{1+c \alpha}\right)
$$

and the (negative-valued) amount of the undershoot

$$
X_{\text {under }}\left(\lambda_{0}\right)=x\left(T_{\text {under }}\left(\lambda_{0}\right)\right)-x(0)
$$


as

$$
\begin{aligned}
X_{\text {under }}\left(\lambda_{0}\right) \\
=\frac{\lambda_{0}^{2}}{8 \omega^{3} \alpha^{2}}\left\{\left(\omega^{3} \alpha+\omega c+\omega \alpha c^{2}\right) t\right. \\
\quad+(1+2 \alpha c) \omega \sin ^{2}(\omega t) \\
\left.\quad+\left(\omega^{2} \alpha-c-\alpha c^{2}\right) \cos (\omega t) \sin (\omega t)\right\}\left.\right|_{t=\Delta t_{A B}\left(\lambda_{0}\right)} \\
\quad+\frac{1}{c}\left\{y_{B}\left(\lambda_{0}\right)+\alpha+\left(\alpha+\frac{1}{c}\right)\right. \\
\left.\quad \times \ln \left(\frac{1-c y_{B}\left(\lambda_{0}\right)}{1+c \alpha}\right)\right\}
\end{aligned}
$$

where $\omega, \Delta t_{A B}$, and $y_{B}\left(\lambda_{0}\right)$ are defined in (70), (71), and (60).

In the case of $\mathrm{P} 2$, the undershoot corresponds to the arc, $F \rightarrow$ $(-\alpha, \bar{\lambda})$ for some $\bar{\lambda}$ in Fig. 7(b) where $(-\alpha, \bar{\lambda})$ lies between $F$ and $G$. One can compute the duration $T_{\text {under }}\left(\lambda_{0}\right)$ of the undershoot as

$$
T=\frac{1}{c} \ln \left(\frac{1}{1+\alpha c}\right)
$$

and the amount $X_{\text {under }}\left(\lambda_{0}\right)$ of the undershoot as

$$
X_{\text {under }}\left(\lambda_{0}\right)=\frac{1}{c}\left(\alpha+\left(\alpha+\frac{1}{c}\right) \ln \left(\frac{1}{1+\alpha c}\right)\right) .
$$

Notice that this undershoot is independent of $\lambda_{0}$.

\section{H. Discussion on State Constraints on $x$}

We have seen in Section III that physics imposes a constraint on $x$ as either

$$
x \geq 0 \text { or } \quad x \leq 0 .
$$

We have not considered these constraints in computing optimal trajectories in this paper. We now make a remark that in some cases optimal trajectories derived without the state constraints are also optimal with the state constraints.

First, let us consider the result in Section IV again-this time with the state constraints in (83). In either case of (83), it is not hard to verify that the result in Section IV still holds without violating the constraint. Namely, there are no time optimal trajectories for $x_{f}<x_{0}$ even in the existence of either state constraint in (83).

Second, we consider the constraint $x \leq 0$ with the initial and final condition satisfying $x_{f} \geq x_{0}$. It is trivial to see that an optimal trajectory derived without the state constraint reaches $x_{f}$ without violating the constraint, so it is still optimal in the existence of the state constraint.

Lastly, we consider the constraint $x \geq 0$ with the initial and final condition satisfying $x_{f} \geq x_{0}$. We divide the discussion into the two cases; $(3+4 \alpha c) \geq 0$ and $(3+4 \alpha c)<0$. When $(3+4 \alpha c) \geq 0$, we know from Section VI-F that there exists a unique $\lambda_{0} \in \Lambda$ such that the two basic arcs $\Gamma_{ \pm}^{\lambda_{0}}$ are the only optimal trajectories. Recall the discussion on initial undershoots in Section VI.G. Also, recall from Section VI-D that $X_{1}$ is a positive function. We have $\dot{x}(t)>0$ after $x(t)$ passes through $x_{0}+X_{\text {under }}\left(\lambda_{0}\right)$ along the basic arc. Hence, we have $\min _{t \in\left[0, T_{1}\left(\lambda_{0}\right)\right]} x(t)=x_{0}+X_{\text {under }}\left(\lambda_{0}\right)$ where $T_{1}\left(\lambda_{0}\right)$ is the duration of the basic arc. It follows that in the case of $(3+4 \alpha c) \geq 0$ if $x_{0} \geq\left|X_{\text {under }}\left(\lambda_{0}\right)\right|$ then the optimal trajectories derived without the state constraint $x \geq 0$ are optimal with the state constraint. On the other hand, as seen in Section VI-F, when $(3+4 \alpha c)<0$ there can be more than two optimal trajectories for a given $x_{f} \geq x_{0}$. Some of the optimal trajectories can be multishot extremals. On an idling arc, one has $\dot{x}=\left(\lambda^{2}-y^{2}\right) /(-4 \alpha)$. Hence, during the first half of an idling arc $\dot{x}(t) \geq 0$ and during the second half $\dot{x}(t) \leq 0$. Recall that an idling arc does not contribute any net displacement in $x$. Let $t_{1}$ and $t_{2}=t_{1}+T_{\text {idling }}$ be the initial and final time of an idling arc of an optimal trajectory with $\lambda_{0} \in \Lambda$ derived without the state constraint $x \geq 0$. Then, it follows that $\min _{t \in\left[t_{1}, t_{2}\right]} x(t)=x\left(t_{1}\right)=x\left(t_{2}\right) \geq x_{0}$. Considering the decomposition of multishot extremals in (19), one can see that in the case of $(3+4 \alpha c)<0$ if $x_{0} \geq\left|X_{\text {under }}\left(\lambda_{0}\right)\right|$ then the optimal trajectories derived without the state constraint $x \geq 0$ remain optimal with the state constraint. Therefore, irrespective of the sign of $(3+4 \alpha c)$, an optimal trajectory with $\lambda_{0} \in \Lambda$ derived without the state constraint $x \geq 0$ is again optimal with the state constraint if $x_{f}>x_{0} \geq\left|X_{\text {under }}\left(\lambda_{0}\right)\right|$.

\section{Symplectic Reduction Picture}

We cast the analysis used in Sections V and VI in the framework of symplectic reduction theory in order to provide geometric insight into the problem. We refer readers to [1] and [9] for symplectic reduction theory.

Let $G=\mathbb{R}$ be the Lie group acting on $M=\mathbb{R}^{2}=\{(x, y)\}$ by translation in $x$ and on the cotangent bundle $T^{*} M=\mathbb{R}^{4}=$ $\left\{\left(x, y, \lambda_{x}, \lambda_{y}\right)\right\}$ by cotangent lift where $T^{*} M$ is equipped with the canonical symplectic form, $\Omega=\mathrm{d} x \wedge \mathrm{d} \lambda_{x}+\mathrm{d} y \wedge \mathrm{d} \lambda_{y}$. The momentum map $J: T^{*} M \rightarrow \mathbb{R}$ corresponding to the $G$-action is $J\left(x, y, \lambda_{x}, \lambda_{y}\right)=\lambda_{x}$. We have $J^{-1}(\nu) \simeq \mathbb{R}^{3}$ for any $\nu \in \mathbb{R}$, and $J^{-1}(\nu) / G \simeq \mathbb{R}^{2}$. One can construct the symplectic projection $\pi: J^{-1}(\nu) \subset T^{*} M \rightarrow J^{-1}(\nu) / G \simeq \mathbb{R}^{2}$ by $\pi\left(x, y, \nu, \lambda_{y}\right)=\left(y, \lambda_{y}\right)$ where $J^{-1}(\nu) / G$ is given the canonical symplectic form $\omega=\mathrm{d} y \wedge \mathrm{d} \lambda_{y}$. Notice that the Hamiltonian $H$ in (32) is $G$-invariant, that the control $u$ in (38) is $G$-invariant, and that the Hamiltonian $M^{\circ}$ in (33) is also $G$-invariant. Hence, $H$ and $M^{\circ}$ induce reduced Hamiltonians $h$ and $m^{\circ}$ on $J^{-1}(\nu) / G$ as follows:

$$
\begin{aligned}
h\left(y, \lambda_{y}, u\right) & =H\left(x, y, \nu, \lambda_{y}, u\right) \\
& =\lambda_{x} \alpha u^{2}+\left(\lambda_{x} y+\lambda_{y}\right) u-c y \lambda_{y} \\
m^{\circ}\left(y, \lambda_{y}\right) & =M^{\circ}\left(x, y, \nu, \lambda_{y}\right)=\max _{|u| \leq 1} h\left(y, \lambda_{y}, u\right) .
\end{aligned}
$$

Notice that the control $u$ maximizing $H\left(x, y, \nu, \lambda_{y}, u\right)$ is the same as the control maximizing $h\left(y, \lambda_{y}, u\right)$ because the control $u$ maximizing $H\left(x, y, \nu, \lambda_{y}, u\right)$ is $G$-invariant and the group $G$ is abelian. Equations (35) and (37) are the (reduced) dynamics of the reduced Hamiltonian $m^{\circ}$ (or $h$ ) on the based space $\left(J^{-1}(\nu) / G, \omega\right)$, and (34) can be regarded as a reconstruction equation to compute the displacement along the fiber $G=\mathbb{R}$. The map $X_{1}$ constructed in Section VI.D measures this displacement while $(y(t), \lambda(t))$ with $(y(0), \lambda(0)) \in 0 \times \Lambda$ stays in 


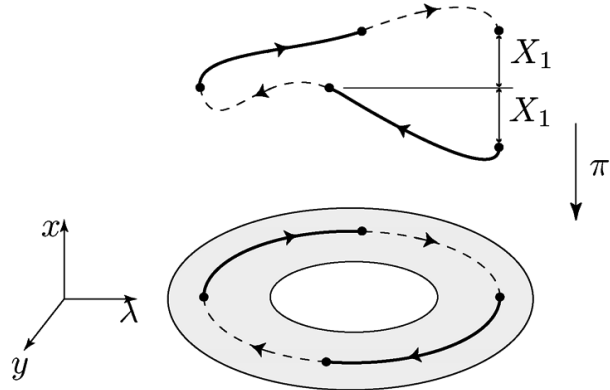

Fig. 11. Level set $J^{-1}(\nu)$ is diffeomorphic to $\mathbb{R}^{3}=\{(x, y, \lambda)\}$ where the $x$-axis is the fiber, and $\pi: J^{-1}(\nu) \rightarrow \mathbb{R}^{2}$ is the projection to the $y$ - $\lambda$ plane. The map $X_{1}$ measures the displacement along the fiber. The dotted arcs are the idling arcs. The shaded region here corresponds to those in Fig. 4.

the first quadrant in the $y$ - $\lambda$ plane. In particular, it is interesting to notice that if $[(1+2 \alpha c) \leq 0]$ or $[(1+2 \alpha c)>0$ and $\lambda(0) \in$ $\Lambda_{1}$ ], then $X_{1}$ measures the half of the total phase (or, holonomy with respect to the trivial connection on the principal bundle $\left.\pi: J^{-1}(\nu) \rightarrow J^{-1}(\nu) / G\right)$ corresponding to a closed trajectory $(y(t), \lambda(t))$ in the base space; see Fig. 11.

In Sections VI.A and VI.B, we did not use the reduced space $\left(\mathbb{R}^{2}, \omega\right)$ and the projection $\pi$ constructed above when $J=\lambda_{x}=$ $\nu \neq 0$, but we used the scaled variable $\lambda=\lambda_{y} / \lambda_{x}$ in (41) and (43) so as to show the discrete symmetry as reflection maps and to deal with all cases of $\lambda_{x}>0$ simultaneously. To put this scaling in the reduction process, one can use $\nu \mathrm{d} y \wedge \mathrm{d} \lambda$ as a symplectic form on the reduced space $J^{-1}(\nu) / G=\{(y, \lambda)\}$, and define the projection $\pi_{\nu}: J^{-1}(\nu) \rightarrow J^{-1}(\nu) / G$ by

$$
\pi_{\nu}:\left(x, y, \nu, \lambda_{y}\right) \mapsto(y, \lambda)=\left(y, \lambda_{y} / \nu\right) .
$$

In this case, the reduced Hamiltonians $h$ and $m^{\circ}$ induced by $H$ and $M^{\circ}$ are those in (84) and (85) with $\lambda_{y}$ replaced by $\nu \lambda$. The vector field $(\dot{y}, \dot{\lambda})$ in Section VI-B is the Hamiltonian vector field of the reduced Hamiltonian $m^{\circ}$ (or $h$ ) on the reduced space $\left(\mathbb{R}^{2}, \nu \mathrm{d} y \wedge \mathrm{d} \lambda\right)$.

Remark 7.1: The $G=\mathbb{R}$ symmetry in the Hamiltonian dynamics was created by the nonlinear coordinate change in (23). This reduction process extends to the case where we consider many particles on the one-dimensional invariant line.

\section{SIMULATIONS}

We demonstrate the theoretical result by simulation. Suppose that the particle and the medium are given such that

$$
\alpha=-3 / 4 \quad c=1
$$

and that the initial and final position of the particle is given by

$$
x_{0}=1 \quad x_{f}=2 .
$$

These numbers are chosen arbitrarily, but one can also do the same simulation with real data once they are given. Let $\Delta x=$ $x_{f}-x_{0}$. One can check that the condition $(1+\alpha c)>0$ for the
TABLE I

Time Costs of the First Five EXtremaLs

\begin{tabular}{|c|c|c|c|}
\hline$n$ & $\lambda_{0, n}$ & $T_{1} \circ X_{1}^{-1}(\Delta x / n)$ & Time cost $T_{n}$ \\
\hline \hline 1 & 0.8649 & 7.8117 & 7.8117 \\
\hline 2 & 0.8577 & 5.7950 & 13.4038 \\
\hline 3 & 0.8491 & 5.1093 & 18.9556 \\
\hline 4 & 0.8415 & 4.7597 & 24.4802 \\
\hline 5 & 0.8350 & 4.5463 & 29.9867 \\
\hline
\end{tabular}

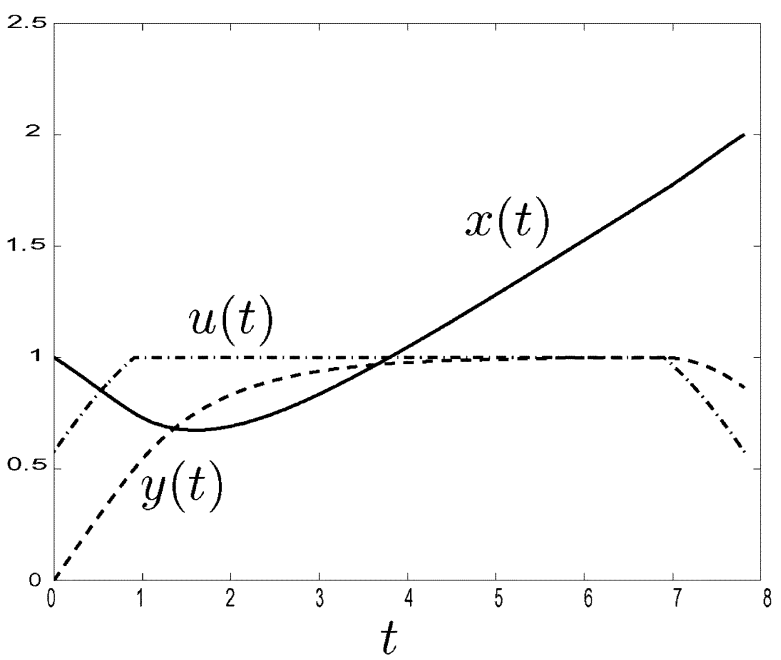

Fig. 12. State $(x(t), y(t))$ of the optimal trajectory and the corresponding optimal control $u(t)$ for $x_{0}=1$ and $x_{f}=2$ with minimum time $t_{f}=7.8117$.

existence of optimal trajectories holds. The idling time in (76) is given by $T_{\text {idling }}=1.8138$. By (63) and (71)-(73), the flight time of the one-shot extremal $T_{1}$ is given by $T_{1}=7.8117$. By the algorithm in Claim 6.5, one learns that the minimum time is in $\left\{T_{1}, \ldots, T_{5}\right\}$. By (82) one computes these flight times in Table I.

Hence, the one-shot extremal is the optimal trajectory and the minimum time is $t_{f}=7.8117$. This result agrees with the uniqueness result because the given $\alpha$ and $c$ satisfy $(3+$ $4 \alpha c) \geq 0$. The optimal control $u(t)$ and the optimal trajectory $(x(t), y(t))$ are given in Fig. 12. Notice the initial undershoot predicted in Section VI-G.

\section{CONCLUSION AND Future Work}

The time-optimal control problem for the dielectrophoretic system studied in this paper has several interesting features. The existence of a term quadratic in control creates the non-existence of optimal trajectories when the final position of the particle is below the initial position. In contrast, when the final position is above the initial position we can show the existence and uniqueness of optimal trajectories in a range of the parameters $\alpha$ and $c$. In the other range of $\alpha$ and $c$ we give a finite algorithm of finding all optimal trajectories instead. Both continuous and discrete symmetry in the problem simplifies the analysis.

In summary, the optimal trajectories are described as follows. There are three different types of optimal trajectories depending on the values of $\alpha$ and $c$, and the displacement of the particle. If $(1+2 \alpha c)>0$, basic arcs, or one-shot extremals, are the 
optimal trajectories. To move a particle a long distance, the optimal control is the saturated controls, i.e., $u= \pm 1$, but for a small displacement of the particle the optimal control consists of three parts; a linear control, a saturated control and a linear control again. If $(1+2 \alpha c) \leq 0$ and $(3+4 \alpha c) \geq 0$, then for any displacement of the particle the optimal control consists of three parts; a linear control, a saturated control and a linear control again. If $(3+4 \alpha c)<0$, optimal trajectories may have the structure of multi-shot extremals in (18) and (19).

As for future work, we will take into account a state constraint, and/or consider the time-optimal control of two different particles for the purpose of separating them which are initially close to each other. They have important applications in nano/ bio-technology [5], [7], [8]. We believe that the use of control systems theory will refine and improve the manipulaton of particles in applications and that our work in this paper makes a first forward step in this direction.

\section{ACKNOWLEDGMENT}

The authors would like to thank B. Bonnard, J. Lévine, J. Marsden, L. Praly, B. Shashikanth, and the anonymous reviewers for invaluable comments.

\section{REFERENCES}

[1] R. Abraham and J. E. Marsden, Foundations of Mechanics, 2nd ed. Reading, MA: Addison-Wesley, 1985, ch. 4, p. 62.

[2] B. Bonnard and M. Chyba, Singular Trajectories and Their Role in Control Theory. New York: Springer-Verlag, 2003.

[3] U. Boscain and B. Piccoli, Optimal Syntheses for Control Systems on 2-D Manifolds. New York: Springer-Verlag, 2004.

[4] D. E. Chang, S. Loire, and I. Mezic, "Closed-form solutions in the electrical field analysis for dielectrophoretic and travelling wave interdigitated electrode arrays," J. Phys. D: Appl. Phys., vol. 36, no. 23, pp. 3073-3078, 2003

[5] D. E. Chang and N. Petit, "Toward controlling dielectrophoresis," Int. J. Robust Nonlinear Control, vol. 15, no. 16, pp. 769-784, 2005

[6] G. de Barra, Measure Theory and Integration. Upper Saddle River, NJ: Prentice-Hall, 1981, pp. 87-88.

[7] M. P. Hughes, Nanoelecromechanics in Engineering and Biology. Boca Raton, FL: CRC, 2002.

[8] T. B. Jones, Electromechanics of Particles. New York: Cambridge Univ. Press, 1995.

[9] J. E. Marsden, R. Montgomery, and T. S. Ratiu, "Reduction, symmetry and phases in mechanics," in AMS Memoirs. Providence, RI: AMS, 1990, vol. 436.

[10] B. Piccoli, "Time-optimal control problems for the swing and ski," Int. J. Control, vol. 62, no. 6, pp. 1409-1429, 1995.

[11] H. A. Pohl, Dielectrophoresis. Cambridge, U.K.: Cambridge Univ. Press, 1978.

[12] L. S. Pontryagin, V. G. Boltyanskii, R. V. Gamkrelidze, and E. F. Mishchenko, The Mathematical Theory of Optimal Processes. New York: Wiley, 1962, pp. 20-50.

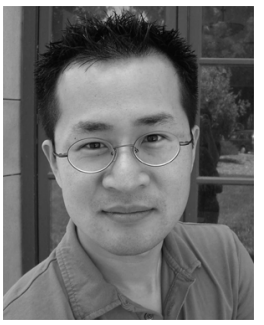

Dong Eui Chang received the B.S. degree in control and instrumentation engineering and the M.S. degree in electrical engineering, both from the Seoul National University, Korea, in 1994 and 1997, respectively, and the Ph.D. degree in control and dynamical systems from the California Institute of Technology, Pasadena, in 2002.

He was a Postdoctoral Fellow at the University of California, Santa Barbara, in 2003, at the Centre Automatique et Systèmes, Ecole Nationale Supérieure des Mines de Paris, France, in 2004, and at the University of Liège, Belgium. He joined the Department of Applied Mathematics at the University of Waterloo, Canada, in August, 2005, as an Assistant Professor. His research interests include geometric control theory and its application to robotics and nanotechnology.

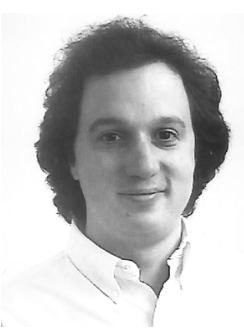

Nicolas Petit was born in Paris, France, in 1972. He graduated from Ecole Polytechnique, Paris, France, in 1995, and received the Ph.D. degree in mathematics and control at Ecole Nationale Supérieure des Mines de Paris, France, in 2000

In 2000-2001, he was a Postdoctoral Scholar in the Control and Dynamical Systems at the California Institute of Technology, Pasadena. Since 2001, he has held the position of Maitre-Assistant at Ecole des Mines de Paris in the Centre Automatique et Systèmes. His research interests include flatness theory for partial differential equations, numerical treatment of optimal trajectory generation problems for nonlinear systems, motion planning, observation of periodic systems, and analysis of distributed systems. On the application side, he is active in industrial process control, engine control, and embedded systems. He has developed the controllers of several industrial chemical reactors, including polystyrene and polypropylene reactors, and the ANAMELV4 and V5 softwares, currently used for closed-loop control of blending devices in numerous refineries. He is a coauthor of several patents in the field of engine control and process control.

Dr. Petit received the Journal of Process Control Paper Prize for Best Article 2002-2005 (Application). He has served as an Associate Editor for Automatica.

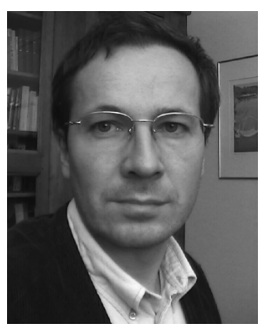

Pierre Rouchon was born in Saint-Etienne, France, in 1960. He graduated from Ecole Polytechnique, Paris, France, in 1983, and received the Ph.D. degree in chemical engineering from Ecole des Mines de Paris, France, in 1990. He received the "habilitation á diriger des recherches" in mathematics from the University Paris-Sud Orsay, France, in 2000.

From 1993 to 2005, he was an Associate Professor at École Polytechnique in Applied Mathematics. From 1998 to 2002, he was the Head of the Centre Automatique et Systèmes of École des Mines de Paris. He is currently a Professor at Ecole des Mines de Paris. His fields of interest include the theory and applications of dynamical systems, nonlinear control, and in particular differential flatness and its extension to infinite-dimensional systems. He has worked on many industrial applications, such as distillation columns, electrical drives, car equipments, and chemical reactors. One of his recent fields of interest is relative to the control and estimation of closed and open quantum systems. 